\title{
MONODROMY OF PARTIAL KZ FUNCTORS FOR RATIONAL CHEREDNIK ALGEBRAS
}

\author{
IAIN G. GORDON AND MAURIZIO MARTINO
}

\section{INTRODUCTION}

1.1. Shan has proved that the categories $\mathcal{O}_{c}\left(W_{n}\right)$ for rational Cherednik algebras of type $W_{n}=$ $W(G(\ell, 1, n))=\mathfrak{S}_{n} \ltimes\left(\mu_{\ell}\right)^{n}$ with $n$ varying, together with decompositions of the parabolic induction and restriction functors of Bezrukavnikov-Etingof, provide a categorification of an integrable $\tilde{\mathfrak{s l}}_{e}$ Fock space representation $\mathcal{F}(\mathbf{m})$, [18]. The parameters $\mathbf{m} \in \mathbb{Z}^{\ell}$ and $e \in \mathbb{N} \cup\{\infty\}$ arise from the choice of parameters $c$ for the rational Cherednik algebra. This categorification gives rise to a crystal structure on the set of irreducible rational Cherednik algebra representations that belong to category $\mathcal{O}_{c}$; it is isomorphic to the crystal introduced by Jimbo-Misra-Miwa-Okado, [11].

The works of many authors, including Kleshchev, Brundan, Lascoux-Leclerc-Thibon, Ariki, Grojnowski-Vazirani, Grojnowski and Chuang-Rouquier, show that the categories $\mathcal{H}_{q}\left(W_{n}\right)$-mod for Hecke algebras of type $W_{n}$ with $n$ varying, together with decompositions of the parabolic induction and restriction functors, provide a categorification of an irreducible integrable $\tilde{\mathfrak{s l}}_{e}$-representation $L(\Lambda)$, [6]. The weight $\Lambda$ arises from the choice of parameters $q$ for the Hecke algebra. This gives rise to a crystal structure on the set of irreducible Hecke algebra representations.

The Fock space is substantially more interesting than the representation $L(\Lambda)$. It is not irreducible, and in fact has an infinite number of non-zero isotypic components. This reducibility reveals itself through distinct canonical bases one can define on $\mathcal{F}(\mathbf{m})$, each of which produces a corresponding crystal. Nonetheless for each $n \in \mathbb{N}$ there is an exact functor $\mathrm{KZ}_{n}: \mathcal{O}_{c}\left(W_{n}\right) \rightarrow \mathcal{H}_{q}\left(W_{n}\right)-\bmod$, [10], which intertwines the parabolic induction and restriction functors for Cherednik algebras and Hecke algebras and produces a compatibility between the corresponding crystals: the component of the Cherednik crystal containing the irreducible representation of $W_{0}=\{1\}$ is isomorphic to the Hecke crystal.

1.2. In this paper we give another construction of a decomposition of the parabolic induction and restriction functors for the rational Cherednik algebra of type an arbitrary complex reflection group. Our construction uses the monodromy of these functors. Together with an appropriate transitivity result for restriction, we explain how these give rise to an $\mathfrak{s}_{e}$-categorification and crystal structure on $\mathcal{F}(\mathbf{m})$ via $\mathcal{O}_{c}\left(W_{n}\right)^{\prime}$ 's. The structure of the proof of these last claims is as in [18. We also

A lot of thanks go to Peng Shan, for many useful discussions spread over a long time. We would also like to thank Ivan Losev for helpful conversations. The first author is grateful for the financial support of EPSRC grant EP/G007632, and also for the hospitality of the Hausdorff Institute for Mathematics in Bonn and ETH in Zürich, where the final writing up was completed. 
show via a homotopy calculation that the decomposition we obtain is naturally isomorphic to the decomposition introduced in [18].

1.3. The only small novelty in our approach is that we do not make use of the double centralizer property of the KZ-functor. It is a fundamental and fruitful technique of [18] to use this property to extend results systematically from Hecke algebras to Cherednik algebras, obtaining in this way definitive results. Optimistically, however, we hope that using the monodromy of the induction and restriction functors alone may be helpful towards generalizations, for instance to Cherednik algebras of varieties with a finite group action, where less is known about the corresponding KZfunctor and where one may imagine branching rules for affine type $B$ and $D$ appear, amongst other things.

1.4. Our results were proved in the second half of 2008, and announced by the first author at the conference "Algebraic Lie Structures with Origins in Physics" at the Isaac Newton Institute in March 2009. It is important to record that although we mentioned then that we knew biadjointness of parabolic restriction and induction, our proof for that turned out to be incomplete. This is one of the most useful results in [18; it is also proved by Losev without use of the KZ-functor, [13]. We use this result here, although it is not needed to obtain the crystal structure. Furthermore we want to say that the presentation of [18] has helped to simplify several of our arguments significantly.

1.5. The outline of the paper is as follows. We recall the restriction and induction functors in Section 2, in both the algebraic and holomorphic settings. In Section 3 we study the monodromy actions on restrictions of modules. Finally in Section 4 we specialize to the $W_{n}$ case to define $i$-restriction and $i$-restriction, and to explain the categorification that then arises. We also check that this does indeed match up with Shan's original results.

\section{Definitions And notation}

2.1. Rational Cherednik algebras. Let $\mathfrak{h}$ be a finite dimensional vector space over $\mathbb{C}$ and $W<$ $G L(\mathfrak{h})$ be a finite subgroup generated by complex reflections. Let $\mathcal{S}$ be the set of complex reflections in $W$ and $\mathcal{A}$ be the corresponding set of reflecting hyperplanes. For each $s \in \mathcal{S}$, let $H_{s}=\operatorname{ker}\left(\alpha_{s}\right)$ denote the reflecting hyperplane of $s$, and define $\alpha_{s}^{\vee} \in \mathfrak{h}$ to be an element such that $\mathfrak{h}=H_{s} \oplus \mathbb{C} \alpha_{s}^{\vee}$ is the $s$-stable decomposition of $\mathfrak{h}$, normalized by $\left\langle\alpha_{s}, \alpha_{s}^{\vee}\right\rangle=2$.

Let $c: \mathcal{S} \rightarrow \mathbb{C}$ be constant on $W$-conjugacy classes. The rational Cherednik algebra attached to $W$ with parameter $c$ is the quotient $H_{c}(W, \mathfrak{h})$ of $T\left(\mathfrak{h} \oplus \mathfrak{h}^{*}\right) \rtimes W$, the smash product of $\mathbb{C} W$ and the tensor algebra of $\mathfrak{h} \oplus \mathfrak{h}^{*}$, by the relations

$$
\left[x, x^{\prime}\right]=0, \quad\left[y, y^{\prime}\right]=0, \quad[y, x]=\langle x, y\rangle-\sum_{s \in \mathcal{S}} c_{s}\left\langle\alpha_{s}, y\right\rangle\left\langle x, \alpha_{s}^{\vee}\right\rangle s,
$$

for all $x, x^{\prime} \in \mathfrak{h}^{*}, y, y^{\prime} \in \mathfrak{h},[8,(1.15)]$.

There is a faithful representation of $H_{c}(W, \mathfrak{h})$ on $\mathbb{C}[\mathfrak{h}]$ where $\mathfrak{h}^{*}$ and $W$ act naturally, and each $y \in \mathfrak{h}$ acts via the Dunkl operator

$$
D_{y}:=\partial_{y}+\sum_{s \in \mathcal{S}} \frac{2 c_{s}}{1-\operatorname{det}_{\mathfrak{h}^{*}}(s)} \frac{\alpha_{s}(y)}{\alpha_{s}}(s-1)
$$


where $\partial_{y}$ is the partial derivative in the direction of $y,[8, \S 4]$. Let $\left\{x_{i}\right\}$ be a basis of $\mathfrak{h}^{*}$ and let $\left\{y_{i}\right\}$ be the dual basis. Then

$$
\mathbf{e u}=\sum_{i} x_{i} y_{i}+\frac{\operatorname{dim}(\mathfrak{h})}{2}-\sum_{s \in \mathcal{S}} \frac{2 c_{s}}{1-\operatorname{det}_{\mathfrak{h}^{*}}(s)} s
$$

is an analogue of the Euler element in $H_{c}(W, \mathfrak{h})$. We have

$$
[\mathbf{e u}, x]=x, \quad[\mathbf{e u}, y]=-y, \quad[\mathbf{e u}, w]=0,
$$

for all $x \in \mathfrak{h}^{*}, y \in \mathfrak{h}$ and $w \in W$.

2.2. Centralizer algebras and the Bezrukavnikov-Etingof isomorphism. Let $b \in \mathfrak{h}$ and let $W_{b} \subset W$ be the stabilizer of $b$ and let $c_{b}$ denote the restriction of the function $c$ to $\mathcal{S} \cap W_{b}$. We write $\mathbb{C}[\mathfrak{h}]^{\wedge} b$ for the completion of $\mathbb{C}[\mathfrak{h}]$ at $b$ in $\mathfrak{h}$, and $\mathbb{C}[\mathfrak{h}]^{\wedge}[b]$ for the completion of $\mathbb{C}[\mathfrak{h}]$ at the $W$-orbit of $b$ in $\mathfrak{h}$. For any $\mathbb{C}[\mathfrak{h}]$-module $M$ let $M^{\wedge} b=\mathbb{C}[\mathfrak{h}]^{\wedge} b \otimes_{\mathbb{C}[\mathfrak{h}]} M$ and $M^{\wedge}[b]=\mathbb{C}[\mathfrak{h}]^{\wedge}[b] \otimes_{\mathbb{C}[\mathfrak{h}]} M$.

The completion $H_{c}(W, \mathfrak{h})^{\wedge}[b]$ can be identified with the subalgebra of $\operatorname{End}_{\mathbb{C}}\left(\mathbb{C}[\mathfrak{h}]^{\wedge}[b]\right)$ generated by $\mathbb{C}[\mathfrak{h}]^{\wedge}[b]$, the Dunkl operators $D_{y}$ for $y \in \mathfrak{h}$, and the group $W$. Let $P=\operatorname{Fun}_{W_{b}}\left(W, H_{c_{b}}\left(W_{b}, \mathfrak{h}\right)^{\wedge} b\right)$ and $Z\left(W, W_{b}, H_{c_{b}}\left(W_{b}, \mathfrak{h}\right)^{\wedge_{b}}\right)$ be the ring of endomorphisms of the right $H_{c_{b}}\left(W_{b}, \mathfrak{h}\right)^{\wedge}{ }^{b}$-module $P$.

Theorem ([3] Theorem 3.2). For any $b \in \mathfrak{h}$ there is an isomorphism of algebras

$$
\Theta_{b}: H_{c}(W, \mathfrak{h})^{\wedge}[b] \longrightarrow Z\left(W, W_{b}, H_{c_{b}}\left(W_{b}, \mathfrak{h}\right)^{\wedge} b\right)
$$

defined as follows: for $f \in P, x \in \mathfrak{h}^{*}, y \in \mathfrak{h}, u, w \in W$,

$$
\begin{aligned}
\left(\Theta_{b}(u) f\right)(w) & =f(w u) \\
\left(\Theta_{b}(x) f\right)(w) & =w(x) f(w) \\
\left(\Theta_{b}(y) f\right)(w) & =w(y) f(w)+\sum_{s \in \mathcal{S} \backslash W_{b}} \frac{2 c_{s}}{1-\operatorname{det}_{\mathfrak{h}^{*}}(s)} \frac{\alpha_{s}(w y)}{x_{\alpha_{s}}}(f(s w)-f(w)) .
\end{aligned}
$$

2.3. Using $\Theta_{b}$ we can identify $H_{c}(W, \mathfrak{h})^{\wedge}[b]$-modules with $\left.Z\left(W, W_{b}, H_{c_{b}}\left(W_{b}, \mathfrak{h}\right)^{\wedge}\right)\right)$-modules. A choice of decomposition of algebras $\mathbb{C}[\mathfrak{h}]^{\wedge}[b] \cong \bigoplus_{p \in W b} \mathbb{C}[\mathfrak{h}]^{\wedge p}$ produces a non-canonical isomorphism of algebras

$$
\Phi: Z\left(W, W_{b}, H_{c_{b}}\left(W_{b}, \mathfrak{h}\right)^{\wedge}{ }^{\wedge}\right) \rightarrow \operatorname{Mat}_{\left|W / W_{b}\right|}\left(H_{c_{b}}\left(W_{b}, \mathfrak{h}\right)^{\wedge}\right)^{\prime}
$$

Let $x_{b} \in \mathbb{C}[\mathfrak{h}]^{\wedge}[b]$ be the idempotent corresponding to 1 under the inclusion $\mathbb{C}[\mathfrak{h}]^{\wedge} \hookrightarrow \mathbb{C}[\mathfrak{h}]^{\wedge}[b]$. Then we can identify $H_{c_{b}}\left(W_{b}, \mathfrak{h}\right)^{\wedge} b$ with $x_{b} H_{c}(W, \mathfrak{h})^{\wedge}{ }^{[b]} x_{b}$. If we denote by $\mathcal{O}_{c}(W, \mathfrak{h})^{\wedge}{ }^{[b]}$ the category of $H_{c}(W, \mathfrak{h})^{\wedge}[b]$-modules that are finitely generated over $\mathbb{C}[\mathfrak{h}]^{\wedge}[b]$, and similarly for $\mathcal{O}_{c_{b}}\left(W_{b}, \mathfrak{h}\right)^{\wedge}$, then there are quasi-inverse equivalences

$$
J: \mathcal{O}_{c_{b}}\left(W_{b}, \mathfrak{h}\right)^{\wedge} \rightarrow \mathcal{O}_{c}(W, \mathfrak{h})^{\wedge}[b], \quad M \mapsto H_{c}(W, \mathfrak{h})^{\wedge}[b] x_{b} \otimes_{H_{c_{b}}\left(W_{b}, \mathfrak{h}\right)^{\wedge}} M
$$

and

$$
R: \mathcal{O}_{c}(W, \mathfrak{h})^{\wedge}[b] \rightarrow \mathcal{O}_{c_{b}}\left(W_{b}, \mathfrak{h}\right)^{\wedge}{ }^{b}, \quad N \mapsto x_{b} N
$$


2.4. Category $\mathcal{O}$ and parabolic restriction and induction. The standard reference for material on category $\mathcal{O}$ is [10]. Category $\mathcal{O}_{c}(W, \mathfrak{h})$ is the full subcategory of the category of $H_{c}(W, \mathfrak{h})$ modules consisting of objects that are finitely generated as $\mathbb{C}[\mathfrak{h}]$-modules and $\mathfrak{h}$-locally nilpotent. A module $M \in \mathcal{O}$ is $\mathfrak{h}$-locally nilpotent if and only if it is eu-locally finite, [3]. The category $\mathcal{O}_{c}(W, \mathfrak{h})$ is a highest weight category. Its standard modules are parametrized by $\operatorname{Irr}(W)$, the irreducible complex representations of $W$ : for any $\lambda \in \operatorname{Irr}(W)$ set $\Delta(\lambda)=H_{c}(W, \mathfrak{h}) \otimes_{\mathbb{C}\left[\mathfrak{h}^{*}\right] \rtimes \mathbb{C} W} \lambda$ where $\mathfrak{h} \subset \mathbb{C}\left[\mathfrak{h}^{*}\right]$ acts by zero on $\lambda$. We denote the irreducible head of $\Delta(\lambda)$ by $L(\lambda)$.

2.5. Parabolic restriction and induction functors were defined in $[3, \S 2.3]$. Let $b \in \mathfrak{h}$ with stabilizer $W_{b}$ and set $\mathfrak{h}_{b}=\mathfrak{h} / \mathfrak{h}^{W_{b}}$. There is an adjoint pair of exact functors $\left((\cdot)^{\wedge}[b], E^{b}\right)$ defined by

$$
(\cdot)^{\wedge}[b]: \mathcal{O}_{c}(W, \mathfrak{h}) \rightarrow \mathcal{O}_{c}(W, \mathfrak{h})^{\wedge[b]}, \quad M \mapsto M^{\wedge[b]} ; \quad E^{b}: \mathcal{O}_{c}(W, \mathfrak{h})^{\wedge}[b] \rightarrow \mathcal{O}_{c}(W, \mathfrak{h}), \quad N \mapsto N^{\mathbf{e u}},
$$

where $N^{\text {eu }} \subset N$ denotes the locally finite part of $N$ under the action of $\mathbf{e u}$, or equivalently the locally nilpotent part of $N$ under the action of $\mathfrak{h}$. If $W_{b}=W$ these are quasi-inverse equivalences. Furthermore there is an equivalence of categories

$$
\zeta: \mathcal{O}_{c_{b}}\left(W_{b}, \mathfrak{h}\right) \rightarrow \mathcal{O}_{c_{b}}\left(W_{b}, \mathfrak{h}_{b}\right), \quad M \mapsto\left\{v \in M: y v=0 \text { for all } y \in \mathfrak{h}^{W_{b}}\right\}
$$

with quasi-inverse

$$
\zeta^{-1}: \mathcal{O}_{c_{b}}\left(W_{b}, \mathfrak{h}_{b}\right) \rightarrow \mathcal{O}_{c_{b}}\left(W_{b}, \mathfrak{h}\right), \quad N \mapsto N \otimes \mathbb{C}\left[\mathfrak{h}^{W_{b}}\right]
$$

where $\mathbb{C}\left[\mathfrak{h}^{W_{b}}\right]$ is the polynomial representation of the ring of polynomial differential operators $\mathcal{D}_{\text {pol }}\left(\mathfrak{h}^{W_{b}}\right)$.

The parabolic restriction and induction functors are then defined as follows, [3, §3.5]:

$$
\begin{aligned}
& \operatorname{Res}_{b}: \mathcal{O}_{c}(W, \mathfrak{h}) \rightarrow \mathcal{O}_{c_{b}}\left(W_{b}, \mathfrak{h}_{b}\right), \quad M \mapsto \zeta \circ E^{b} \circ R\left(M^{\wedge}[b]\right), \\
& \operatorname{Ind}_{b}: \mathcal{O}_{c_{b}}\left(W_{b}, \mathfrak{h}_{b}\right) \rightarrow \mathcal{O}_{c}(W, \mathfrak{h}), \quad N \mapsto E^{b} \circ J\left(\zeta^{-1}(N)^{\wedge}\right) .
\end{aligned}
$$

By [3, Theorem 3.10] $\operatorname{Ind}_{b}$ is right adjoint to $\operatorname{Res}_{b}$.

2.6. Basechange. Let $S$ denote the formal power series ring $\mathbb{C}\left[\left[\bar{c}_{s}-c_{s}\right]\right]_{s \in \mathcal{S}}$, where the $\bar{c}_{s}$ denote indeterminates such that $\bar{c}_{s}=\bar{c}_{s^{\prime}}$ if $s$ and $s^{\prime}$ are conjugate in $W$. We denote by $\mathfrak{m}$ the maximal ideal of $S$ and let $K$ be the quotient field of $S$. The rational Cherednik algebras over these base rings, defined at the formal parameters $\bar{c}_{s}$, are denoted by $H_{S}(W, \mathfrak{h})$ and $H_{K}(W, \mathfrak{h})$ respectively.

The definition of category $\mathcal{O}$ makes sense over $K$, and is denoted $\mathcal{O}_{K}(W, \mathfrak{h})$. This is a semisimple category whose simple objects are given by the standard modules $\Delta_{K}(\lambda)$ for $\lambda \in \operatorname{Irr}(W)$. We can also construct standard modules over $S, \Delta_{S}(\lambda)$, and we define $\mathcal{O}_{S}^{\Delta}(W, \mathfrak{h})$ to be the full subcategory of finitely generated $H_{S}(W, \mathfrak{h})$-modules that are free as $S[\mathfrak{h}]$-modules. This is motivated by [10, Proposition 2.21] which states that the objects of $\mathcal{O}_{c}^{\Delta}(W, \mathfrak{h})$, the full subcategory of $\mathcal{O}_{c}(W, \mathfrak{h})$ consisting of modules with filtrations by standard modules, are precisely those for which the action of $\mathbb{C}[\mathfrak{h}]$ is free. 
2.7. All of the preceding constructions are well-defined over $S$ and $K$. Thus we can define restriction functors $\operatorname{Res}_{b, S}$ and $\operatorname{Res}_{b, K}$.

Lemma. The following diagram commutes:

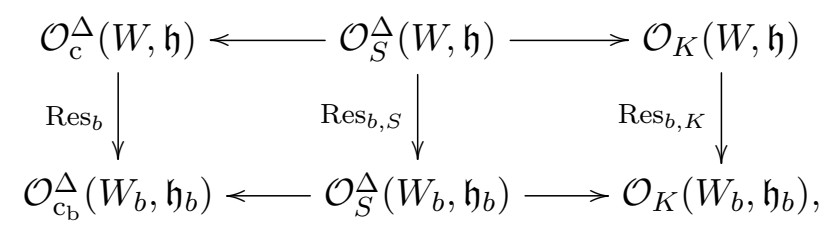

where the arrows to the left denote $-\otimes_{S} S / \mathfrak{m}$ and arrows to the right denote $-\otimes_{S} K$.

Proof. Let $Z_{S}=Z\left(W, W_{b}, H_{S}\left(W_{b}, \mathfrak{h}\right)^{\wedge} b\right)$ and $Z_{K}=Z\left(W, W_{b}, H_{K}\left(W_{b}, \mathfrak{h}\right)^{\wedge} b\right)$. There is a commutative diagram of homomorphisms:

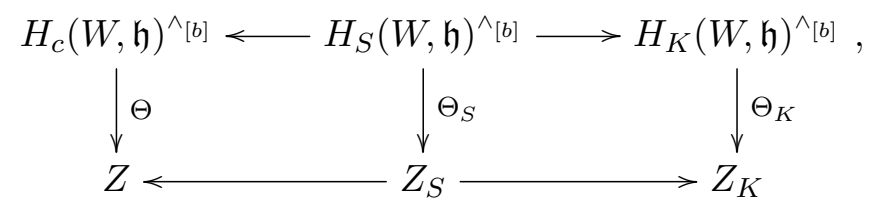

where the horizontal arrows denote the homomorphisms corresponding to the natural maps $S \rightarrow$ $S / \mathfrak{m}$ and $S \rightarrow K$.

Let $M \in \mathcal{O}_{S}^{\Delta}(W, \mathfrak{h})$. We claim that the idempotent $x_{b}$ lifts uniquely from $Z$ to $Z_{S}$. Indeed, the isomorphism $\Phi$ is derived from a choice of decomposition $\mathbb{C}[\mathfrak{h}]^{\wedge}[b] \cong \prod_{p \in W \cdot b} \mathbb{C}[\mathfrak{h}]^{\wedge p}$. Let $\mathfrak{n} \subset \mathbb{C}[\mathfrak{h}]$ denote the ideal of functions vanishing on $W \cdot b$. Then by the unique lifting property for complete rings we can lift $\left(x_{b}\right)_{n}$ uniquely to $S[\mathfrak{h}] /(\mathfrak{n})^{n}$ along the homomorphism $S[\mathfrak{h}] /(\mathfrak{n})^{n} \rightarrow \mathbb{C}[\mathfrak{h}] /(\mathfrak{n})^{n}$, where $\left(x_{b}\right)_{n}$ denotes the image of $x_{b}$ in $\mathbb{C}[\mathfrak{h}]^{\wedge}[b] /(\mathfrak{n})^{n}$. Therefore, $x_{b}$ lifts uniquely to $S[\mathfrak{h}]^{\wedge}[b]$. The claim now follows. As a consequence of this lifting, we have $R_{S}\left(M^{\wedge}[b]\right) \otimes_{S} S / \mathfrak{m} \cong R\left(M^{\wedge}[b] \otimes_{S} S / \mathfrak{m}\right)$.

We show that the left hand square of (1) commutes by establishing that

$$
\left(E_{S} \circ R_{S}\left(M^{\wedge}[b]\right)\right) \otimes_{S} S / \mathfrak{m} \cong E \circ R\left(M^{\wedge}[b]\right) .
$$

The module $N=R_{S}\left(M^{\wedge}[b]\right)$ is a finitely generated $S[\mathfrak{h}]^{\wedge}[b]$-module. Let $m$ be the ideal of positive degree formal power series, and let $\sim$ denote the completion with respect to $m$. By the proof of [3. Theorem 2.3], which is valid over $S$, we have $\widetilde{E_{S}(N)}=N$. In particular, there exists a set $n_{1}, \ldots, n_{l} \in N$ consisting of generalized eu-eigenvectors that generate $N$ as an $S[\mathfrak{h}]^{\wedge}[b]$-module. Let us denote by $\bar{n}_{i}$ the image of $n_{i}$ in $N \otimes_{S} S / \mathfrak{m}$. By [3, Theorem 2.3] again, $E_{S}(N)=S[\mathfrak{h}] n_{1}+\cdots+$ $S[\mathfrak{h}] n_{l}$ and so $E_{S}(N) \otimes_{S} S / \mathfrak{m}=\mathbb{C}[\mathfrak{h}] \bar{n}_{1}+\cdots+\mathbb{C}[\mathfrak{h}] \bar{n}_{l}$. On the other hand, by the previous paragraph, $N \otimes_{S} S / \mathfrak{m}=\mathbb{C}[\mathfrak{h}]^{\wedge}[b] \bar{n}_{1}+\cdots+\mathbb{C}[\mathfrak{h}]^{\wedge}[b] \bar{n}_{l}$, and so $E\left(N \otimes_{S} S / \mathfrak{m}\right)=\mathbb{C}[\mathfrak{h}] \bar{n}_{1}+\cdots+\mathbb{C}[\mathfrak{h}] \bar{n}_{l}=E_{S}(N) \otimes_{S} \mathbb{C}$.

It remains to show that the right-hand square of (1) commutes. It is clear that

$$
\left(R_{S}\left(M^{\wedge}[b]\right)\right) \otimes_{S} K \cong R_{K}\left(M^{\wedge}[b]\right) .
$$

By the arguments above, $N=R_{S}\left(M^{\wedge}[b]\right)$ is generated by a finite set of generalized eu-eigenvectors. Therefore $\left\{n \in N \otimes_{S} K: n\right.$ is locally finite for $\left.\mathbf{e u}\right\}$ is the image of the set of eu-finite vectors in $N$ by the functor $-\otimes_{S} K$. 
2.8. Holomorphic version. Let $X$ be a complex manifold and define $\mathcal{K}_{X}$ to be the sheaf of holomorphic functions on $X$. For $x \in X$, we denote by $\mathcal{K}_{X, x}$ the germs of holomorphic functions around $x$. We also denote by $\widehat{\mathcal{K}}_{X, x}$ the algebra of formal series in local coordinates around $x$. There is an injective algebra homomorphism $\mathcal{K}_{X, x} \rightarrow \widehat{\mathcal{K}}_{X, x}$, sending a germ to its Maclaurin series around $x$. Given a sheaf $\mathcal{M}$ of $\mathcal{K}_{X}$-modules, we denote its stalk at $x$ by $\mathcal{M}_{x}$ and define $\mathcal{M}^{\wedge x}$ to be $\widehat{\mathcal{K}}_{X, x} \otimes \mathcal{K}_{X, x} \mathcal{M}_{x}$.

2.9. We now consider $\mathfrak{h}$ with the complex topology. Let $U \subseteq \mathfrak{h}$ be a connected $W$-stable open subset containing $b$. Define $\left.H_{c}(W)\right|_{U}$ to be the sheaf of algebras on $U / W$ whose sections at a $W$ invariant open subset $V \subseteq U$ are the subalgebra of $\operatorname{End}_{\mathbb{C}}\left(\mathcal{K}_{U}(V)\right)$ generated by $W, \mathcal{K}_{U}(V)$ and the Dunkl operators $D_{y}$ for $y \in \mathfrak{h}$. For a $W$-stable open subset $V \subseteq U$, let $H_{c}(W, V)=\left.H_{c}(W)\right|_{U}(V)$.

Let $W^{\prime} \subseteq W$ be a subgroup, not necessarily parabolic. Let $U \subseteq \mathfrak{h}$ be an $W^{\prime}$-stable open subset such that

$$
w \cdot U \cap U=\emptyset, \text { for } w \in W \backslash W^{\prime}
$$

Thus $W \cdot U=\bigsqcup_{w \in W / W^{\prime}} w \cdot U$. Let $c^{\prime}$ denote the restriction of $c$ to $\mathcal{S} \cap W^{\prime}$. For each $y \in \mathfrak{h}$ define Dunkl operators

$$
D_{y}^{\prime}:=\partial_{y}+\sum_{s \in \mathcal{S} \cap W^{\prime}} \frac{2 c_{s}}{1-\operatorname{det}_{\mathfrak{h}^{*}}(s)} \frac{\alpha_{s}(y)}{\alpha_{s}}(s-1) .
$$

As above, we can define a sheaf of algebras on $U / W^{\prime}$, which we denote $\left.H_{c^{\prime}}\left(W^{\prime}\right)\right|_{U}$. The endomorphisms of the sheaf $\operatorname{Fun}_{W^{\prime}}\left(W,\left.H_{C^{\prime}}(W)\right|_{U}\right)$ form a sheaf of algebras $Z\left(W, W^{\prime},\left.H_{c^{\prime}}\left(W^{\prime}\right)\right|_{U}\right)$.

Theorem ([3]). Let $W^{\prime}$ and $U$ be as above. Then there is an isomorphism of sheaves of algebras

$$
\Theta_{U}:\left.H_{c}(W)\right|_{W \cdot U} \longrightarrow Z\left(W, W^{\prime},\left.H_{c^{\prime}}\left(W^{\prime}\right)\right|_{U}\right)
$$

which is given as follows. Let $f$ be a section of $\operatorname{Fun}_{W^{\prime}}\left(W,\left.H_{c}(W)\right|_{W \cdot U}\right)$, then

$$
\begin{aligned}
& \left(\Theta_{U}(u) f\right)(w)=f(w u), \\
& \left(\Theta_{U}(\phi) f\right)(w)=\left(\left.\phi^{w}\right|_{V}\right) f(w), \\
& \left(\Theta_{U}(y) f\right)(w)=w(y) f(w)+\sum_{s \in \mathcal{S}, s \notin W^{\prime}} \frac{2 c_{s}}{1-\operatorname{det}_{\mathfrak{h}^{*}}(s)} \frac{\alpha_{s}(w y)}{x_{\alpha_{s}}}(f(s w)-f(w)),
\end{aligned}
$$

where $u, w \in W, \phi$ is a section of $\mathcal{K}_{W \cdot U}$ and $y \in \mathfrak{h}$.

2.10. Let $\mathcal{O}_{c}(W, W \cdot U)$ be the category of $\left.H_{c}(W)\right|_{W \cdot U}$-modules that are coherent as $\mathcal{K}_{W \cdot U}$-modules. Letting $1_{U}$ play the analogous role to $x_{b}$ in 2.3 , there are quasi-inverse equivalences

$$
J_{U}: \mathcal{O}_{c^{\prime}}\left(W^{\prime}, U\right) \rightarrow \mathcal{O}_{c}(W, W \cdot U), \quad \mathcal{M} \mapsto H_{c}(W)_{W \cdot U} 1_{U} \otimes_{H_{c^{\prime}}\left(W^{\prime}\right)_{U}} \mathcal{M}
$$

and

$$
R_{U}: \mathcal{O}_{c}(W, W \cdot U) \rightarrow \mathcal{O}_{c^{\prime}}\left(W^{\prime}, U\right), \quad \mathcal{N} \mapsto 1_{U} \mathcal{N}
$$


2.11. Let $\mathcal{M}$ be a sheaf on $V \subseteq \mathfrak{h}$, where $V$ is an open set containing the orbit $W \cdot b$. Then we can define $\mathcal{M}^{\wedge}[b]=\bigoplus_{p \in W \cdot b} \mathcal{M}^{\wedge p}$.

Lemma. Let $M \in \mathcal{O}_{c}(W, \mathfrak{h})$ and set $\mathcal{M}=\mathcal{K}_{\mathfrak{h}} \otimes_{\mathbb{C}[\mathfrak{h}]} M$. Let $U \subset \mathfrak{h}$ and $W^{\prime} \subseteq W$ be as above, and assume that $b \in U$ has stabilizer $W_{b}$ contained in $W^{\prime}$. Then there are natural isomorphisms of $H_{c_{b}}\left(W_{b}, \mathfrak{h}\right)^{\wedge} b-$ modules:

$$
R\left(M^{\wedge}[b]\right) \cong R\left(\left(\left.\mathcal{M}\right|_{W \cdot U}\right)^{\wedge}[b]\right) \cong R\left(\left(R_{U}\left(\left.\mathcal{M}\right|_{W \cdot U}\right)\right)^{\wedge} W^{\prime} \cdot b\right) .
$$

Proof. The first isomorphism is clear since $M^{\wedge}[b] \cong\left(\left.\mathcal{M}\right|_{W \cdot U}\right)^{\wedge}[b]$. For the second isomorphism we can find a connected $W_{b^{-}}$stable open subset $V$ of $U$ containing $b$ such that $w \cdot V \cap V=\emptyset$ for $w \in W^{\prime} \backslash W_{b}$. Let $\mathcal{N}=R_{U}\left(\left.\mathcal{M}\right|_{W \cdot U}\right)$. We have $R\left(\mathcal{N}^{\wedge} W^{\prime} \cdot b\right) \cong R\left(\left(\left.\mathcal{N}\right|_{W^{\prime} \cdot V}\right)^{\wedge} W^{\prime} \cdot b\right)$. There is a decomposition

$$
\left.\left.\mathcal{N}\right|_{W^{\prime} \cdot V} \cong \bigoplus_{w \in W^{\prime} / W_{b}} \mathcal{N}\right|_{w \cdot V}
$$

such that $R_{V}\left(\left.\mathcal{N}\right|_{W^{\prime} \cdot V}\right)$ is the projection onto $\left.\mathcal{N}\right|_{V}$, and we have a commutative diagram

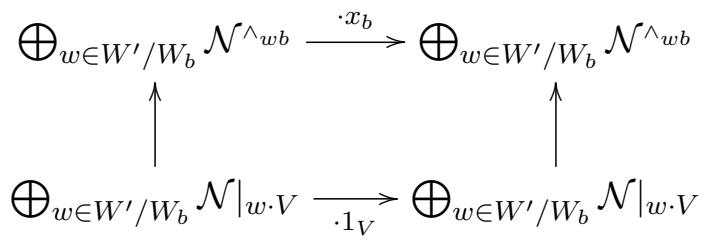

where the vertical arrows denote the canonical morphisms into completions. Thus $R\left(\left(\left.\mathcal{N}\right|_{W^{\prime} \cdot V}\right)^{\wedge} W^{\prime} \cdot b\right) \cong$ $\left(R_{V}\left(\left.\mathcal{N}\right|_{W^{\prime} \cdot V}\right)\right)^{\wedge}$. We have $1_{V} 1_{U}=1_{V}$ on $W \cdot V$, so that $R_{V}\left(\left.\mathcal{N}\right|_{W^{\prime} \cdot V}\right) \cong R_{V}\left(\left.\mathcal{M}\right|_{W \cdot V}\right)$. Hence, using for the second isomorphism below the same commutative diagram logic, we deduce

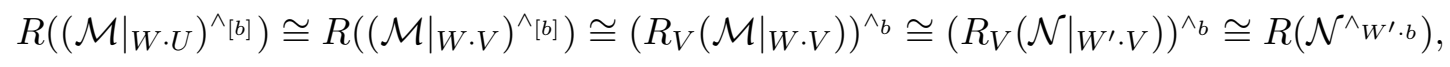

as required.

2.12. We define

$$
\operatorname{Res}_{b, U}: \mathcal{O}_{c}(W, \mathfrak{h}) \rightarrow \mathcal{O}_{c_{b}}\left(W_{b}, \mathfrak{h}_{b}\right), \quad M \mapsto \zeta \circ E^{b} \circ R\left(\left(R_{U}\left(\left.\mathcal{M}\right|_{W \cdot U}\right)\right)^{\wedge} W^{\prime \cdot b}\right) .
$$

It then follows from Lemma 2.11 that

Corollary. The functors $\operatorname{Res}_{b, U}$ and $\operatorname{Res}_{b}$ are naturally isomorphic.

2.13. Now note that the module $R_{U}\left(\left.\mathcal{M}\right|_{W \cdot U}\right)$ has an action of $W^{\prime}$, since $U$ is $W^{\prime}$-stable. Assume that $W^{\prime}$ normalizes $W_{b}$. For each $w \in W^{\prime}$, there is a well-defined automorphism of $H_{c_{b}}\left(W_{b}, \mathfrak{h}_{b}\right)$ given by $a \mapsto w a w^{-1}$ for all $a \in H_{c_{b}}\left(W_{b}, \mathfrak{h}_{b}\right)$. Similarly, we obtain an isomorphism $x_{b} H_{c}(W, \mathfrak{h})^{\wedge}[b] x_{b} \cong$ $x_{w b} H_{c}(W, \mathfrak{h})^{\wedge}[b] x_{w b}$ via conjugation. There is a commutative diagram of algebra homomorphisms

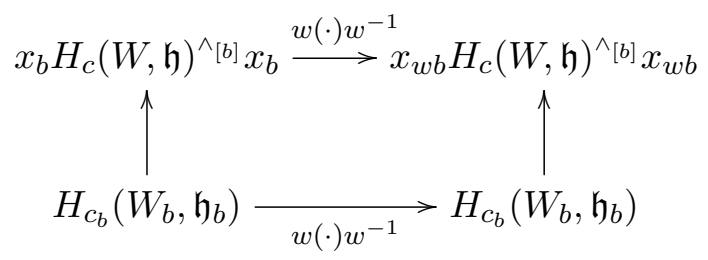

where the vertical arrow denote inclusion maps. 
Lemma. Let $w \in W^{\prime}$ and let $N \in \mathcal{O}_{c}(W, \mathfrak{h})^{\wedge}[b]$. Then, as an $H_{c_{b}}\left(W_{b}, \mathfrak{h}_{b}\right)$-module, $x_{w b} N$ is isomorphic to the twist of $x_{b} N$ by the automorphism $w(\cdot) w^{-1}$. In particular, if $w$ centralizes $H_{c_{b}}\left(W_{b}, \mathfrak{h}_{b}\right)$, then these modules are isomorphic.

\section{Monodromy aCtions}

3.1. Fundamental groups. Let us fix throughout a $W$-invariant Hermitian form $(-,-)$ on $\mathfrak{h}$, and let $\|\cdot\|$ be the associated Hermitian product. Let $b \in \mathfrak{h}, W_{b} \subseteq W$ the stabilizer of $b$, and $W^{\prime}=N_{W}\left(W_{b}\right)$ be the normalizer of $W_{b}$ in $W$. Let $\mathfrak{h}^{W_{b}}$ the fixed point set and $\mathfrak{h}_{b}$ the orthogonal complement to $\mathfrak{h}^{W_{b}}$ in $\mathfrak{h}$. Note that the decomposition $\mathfrak{h}=\mathfrak{h}^{W_{b}} \oplus \mathfrak{h}_{b}$ is $W^{\prime}$-stable. If we wish to consider $b$ as an element of $\mathfrak{h}^{W_{b}}$ we will write it as $b^{\prime}$, so that $b=\left(b^{\prime}, 0\right) \in \mathfrak{h}^{W_{b}} \oplus \mathfrak{h}_{b}$.

Proposition ([14]). There is a subgroup $N \subseteq W^{\prime}$ such that $N \cap W_{b}=1$ and $W^{\prime}=W_{b} \rtimes N$.

This proposition allows us to define $C \subseteq N$ to be the pointwise stabilizer of $\mathfrak{h}_{b}$. In particular $C$ centralizes $W_{b}$.

3.2. Let $\mathcal{S}_{b}=\mathcal{S} \cap W_{b}$ and $\mathcal{A}_{b} \subseteq \mathcal{A}$ denote the corresponding reflecting hyperplanes. We define $\mathcal{S}^{\prime}$ and $\mathcal{A}^{\prime}$ similary, using $W^{\prime}$. Let

$$
\mathfrak{h}_{\mathrm{r}}^{W_{b}}=\left\{x \in \mathfrak{h}: W_{x}=W_{b}\right\} .
$$

Let $B_{C}=\pi_{1}\left(\mathfrak{h}_{\mathrm{r}}^{W_{b}} / C, b^{\prime}\right)$ and $B_{N}=\pi_{1}\left(\mathfrak{h}_{\mathrm{r}}^{W_{b}} / N, b^{\prime}\right)$, where we abuse notation by letting $b^{\prime}$ denote the image of $b^{\prime} \in \mathfrak{h}_{\mathrm{r}}^{W_{b}}$ in the relevant orbit space. Since $\mathfrak{h}_{\mathrm{r}}^{W_{b}} / C \rightarrow \mathfrak{h}_{\mathrm{r}}^{W_{b}} / N$ is a normal covering, we have an exact sequence

$$
1 \longrightarrow B_{C} \longrightarrow B_{N} \stackrel{\beta}{\longrightarrow} N / C \longrightarrow 1 \text {. }
$$

Note that in the special case that $b$ is generic, we have $W_{b}=1, N=W$ and $B_{W}$ is the braid group attached to $(W, \mathfrak{h})$.

3.3. Let $\epsilon>0$ be a real number. Let $X$ denote the open ball of radius $\epsilon$ around 0 in $\mathfrak{h}_{b}$. For any $v \in \mathfrak{h}^{W_{b}}$ we let $\mathcal{B}_{v}(\epsilon)$ denote the open ball in $\mathfrak{h}^{W_{b}}$ with centre $v$ and radius $\epsilon$. Take the annulus

$$
Y=\bigcup_{v \in \mathfrak{h}^{W_{b}} ;\|v\|=\left\|b^{\prime}\right\|} \mathcal{B}_{v}(\epsilon),
$$

and let $Y_{\mathrm{r}}=Y \cap \mathfrak{h}_{\mathrm{r}}^{W_{b}}$. Set $U=X \times Y_{\mathrm{r}}$. This open subset is $W^{\prime}$-stable, and we choose $\epsilon$ small enough so that $w \cdot U \cap U=\emptyset$ for all $w \in W \backslash W^{\prime}$. In particular, $U$ intersects only the reflecting hyperplanes in $\mathcal{A}^{b}$. Since $Y_{\mathrm{r}}$ is homotopic to $\mathfrak{h}_{\mathrm{r}}^{W_{b}}$ and $X$ is simply connected we have

$$
\pi_{1}(U / N, b) \cong \pi_{1}\left(\mathfrak{h}_{\mathrm{r}}^{W_{b}} / N, b^{\prime}\right)=B_{N}
$$

3.4. Holomorphic differential operators. Given a complex manifold $V$, let $\mathcal{D}_{V}$ denote the sheaf of holomorphic differential operators on $V$.

Lemma. Keep the above notation. Let $p_{1}: U \rightarrow X$ and $p_{2}: U \rightarrow Y_{r}$ be the projections. The sheaf $\left.H_{c^{\prime}}\left(W^{\prime}, \mathfrak{h}\right)\right|_{U}$ contains subsheaves $\left.p_{1}^{-1} H_{c^{\prime}}\left(W_{b}, \mathfrak{h}_{b}\right)\right|_{X}$ and $p_{2}^{-1}\left(\mathcal{D}_{Y_{r}} \rtimes N\right)$. Furthermore $\left.p_{1}^{-1} H_{c^{\prime}}\left(W_{b}, \mathfrak{h}_{b}\right)\right|_{X}$ and $p_{2}^{-1}\left(\mathcal{D}_{Y_{r}}\right)$ commute. 
Proof. Let $V \subseteq U$ be an $W^{\prime}$-stable open subset. By our assumptions on $Y_{\mathrm{r}}$ and $X$, the functions $\frac{1}{\alpha_{s}}$ for $s \in \mathcal{S} \backslash W_{b}$ are well-defined on all of $U$. So $H_{c^{\prime}}\left(W^{\prime}, V\right)$ contains the operators

$$
D_{y}^{\prime}=D_{y}-\sum_{s \in \mathcal{S}, s \notin W_{b}} \frac{2 c_{s}}{1-\operatorname{det}_{\mathfrak{h}^{*}}(s)} \frac{\alpha_{s}(y)}{\alpha_{s}}(s-1),
$$

defined in 2.9 for all $y \in \mathfrak{h}$. The subalgebra generated by $\mathcal{K}_{X}\left(p_{1}(V)\right), W_{b}$ and $D_{y}^{\prime}$ for $y \in \mathfrak{h}_{b}$ generate a copy of $H_{c_{b}}\left(W_{b}, p_{1}(V)\right)$. Similarly, the algebra generated by $\mathcal{K}_{Y_{\mathrm{r}}}\left(p_{2}(V)\right), N$ and the $D_{y}^{\prime}=\partial_{y}$ for $y \in \mathfrak{h}^{W_{b}}$ yield a copy of $\mathcal{D}_{Y_{\mathrm{r}}}\left(p_{2}(V)\right) \rtimes N$. It is straightforward to check that the final assertion holds.

3.5. Monodromy. Let $\lambda \in \operatorname{Irr}(W)$, and let $M=\Delta(\lambda)$ be the corresponding standard module. In the notation of 2.10, we set $\mathcal{N}=R_{U}\left(\left.\mathcal{M}\right|_{W \cdot U}\right)$. By Lemma 3.4, the action of $\partial_{y}, y \in \mathfrak{h}^{W_{b}}$, on $\mathcal{N}(U)$ defines an $N$-equivariant connection on $Y_{\mathrm{r}}$ with parameters in $\mathcal{K}_{X}(X)$, see [19, §13] for information on linear differential equations with parameters.

Proposition ([3], Proposition 3.20). The local system on $Y_{r}$ attached to $\mathcal{N}(U)$ is given by the connection form

$$
\sum_{s \in \mathcal{S} \backslash W_{b}} \frac{2 c_{s}}{1-\operatorname{det}_{\mathfrak{h}^{*}}(s)} \frac{d \alpha_{s}}{\alpha_{s}}(s-1) .
$$

This is a connection with parameters in $\mathcal{K}_{X}(X)$ on the trivial bundle on $Y_{r}$ taking values in $\bigoplus_{\nu \in \operatorname{Irr}\left(W_{b}\right)} \operatorname{Hom}_{W_{b}}\left(\nu, \lambda \downarrow_{W_{b}}\right)$.

We denote this connection by $\nabla^{\lambda}$, and for a pair $(c, p) \in \mathbb{C}^{|\mathcal{S} / W|} \times X$ we denote its specialization to this point as $\nabla_{c, p}^{\lambda}$.

3.6. Let $m=\operatorname{dim} E$. By [16], there exist functions $\phi_{1}, \ldots, \phi_{m}$, holomorphic on $\mathbb{C}^{|\mathcal{S} / W|} \times X \times Y^{\prime}$, where $Y^{\prime} \subset Y_{\mathrm{r}}$ is an open ball containing $b^{\prime}$, such that specialising to a point $(c, p) \in \mathbb{C}^{|\mathcal{S} / W|} \times X$ yields the horizontal sections of $\nabla_{c, p}^{\lambda}$ in $Y^{\prime}$. Since the connection is $N$-equivariant, we can associate to each $g \in B_{N}$ a monodromy matrix acting on the span of the $\phi_{j}$. We call this $g^{\lambda}$ and its specialization $g_{c, p}^{\lambda}$.

Corollary. The stalk at $b^{\prime}$ of the local system $\mathcal{N}^{\nabla^{\lambda}}$ on $Y_{r}$ can be evaluated by taking horizontal sections of $\nabla^{\lambda}$ in either $\mathcal{N}_{b^{\prime}}$ or $\mathcal{N}^{\wedge} b^{\prime}$. In particular, we can identify the completion of $\mathcal{N}_{b^{\prime}}^{\nabla^{\lambda}}$ at $0 \in \mathfrak{h}_{b}$ with $\zeta\left(\operatorname{Res}_{b}(M)\right)^{\wedge}$.

Proof. By the proposition, the connection on $\nabla_{(c, p)}^{E}$ has regular singularities at $b^{\prime}$ for all $(c, p)$. The equality of horizontal sections in the convergent or formal setting is then well-known, see [15] for example. The second claim follows from Corollary 2.12.

3.7. Continue with the above notation.

Lemma. The elements $w \in W_{b}, f \in \widehat{\mathcal{K}}_{X, 0}$ and $D_{y}^{\prime}$ for $y \in \mathfrak{h}_{b}$ act on $\left(\mathcal{N}_{b^{\prime}}^{\nabla^{\lambda}}\right)^{\wedge}$. Let $P$ denote any of these operators. These $P$ commute with $g^{\lambda}$ via $\left(g^{\lambda}\right)^{-1} P g^{\lambda}=\beta(g)(P)$ where $\beta$ is defined in (2). 
Proof. By Lemma 3.4, any $w \in W_{b}, f \in \widehat{\mathcal{K}}_{X, 0}$ and $D_{y}^{\prime}, y \in \mathfrak{h}_{b}$, act on the completion of $\mathcal{N}_{b^{\prime}}$ at $0 \in \mathfrak{h}_{b}$, and commute with the action of $\left(\pi_{2}^{-1} \mathcal{D}_{Y_{\mathrm{r}}}\right)_{b} \cong\left(\mathcal{D}_{Y_{\mathrm{r}}}\right)_{b^{\prime}}$. So the operators certainly act.

Recall that $g^{\lambda}$ is calculated as follows. We represent $g$ by a path $p$ from $b^{\prime}$ to $n b^{\prime}$ for some $n \in N$. Then we let $A_{p}$ denote the analytic continuation operator along $p$, a linear isomorphism $A_{p}: \mathcal{N}_{b^{\prime}}^{\nabla^{\lambda}} \rightarrow \mathcal{N}_{n b^{\prime}}^{\nabla^{\lambda}}$. Then $g^{\lambda}: \mathcal{N}_{b^{\prime}}^{\nabla^{\lambda}} \rightarrow \mathcal{N}_{b^{\prime}}^{\nabla^{\lambda}}$ is given by $n^{-1} A_{p}$.

Let $v_{b^{\prime}} \in \mathcal{N}_{b^{\prime}}^{\nabla_{(c, 0)}^{\prime}}$ and $P$ denote one of the operators $w, f$ or $D_{y}^{\prime}$ as above. Uniqueness of analytic continuation implies that $A_{p}\left(P v_{b^{\prime}}\right)=P\left(A_{p} v_{b^{\prime}}\right)$ and so if $\bar{A}_{p}$ denotes the reverse path to $A_{p}$ we have

$$
\left(g^{E}\right)^{-1} P g^{E}\left(v_{b^{\prime}}\right)=\bar{A}_{p} n\left(P n^{-1}\left(A_{p} v_{b^{\prime}}\right)\right)=\left(\bar{A}_{p} P^{n} A_{p}\right)\left(v_{b^{\prime}}\right)=P^{n} v_{b^{\prime}}=\beta(g)(P) v_{b^{\prime}},
$$

as required.

3.8. Using the short exact sequence (2) we define we define an action of $B_{N}$ on $H_{c^{\prime}}\left(W_{b}, \mathfrak{h}_{b}\right)$ via the quotient $B_{N} / B_{C}$.

Theorem. Let $M \in \mathcal{O}_{c}(W, \mathfrak{h})$. Then $\operatorname{Res}_{b}(M)$ has an action of $H_{c_{b}}\left(W_{b}, \mathfrak{h}_{b}\right) \rtimes B_{N}$. The $B_{N}$-action is functorial.

Proof. Let us first suppose that $M=\Delta(\lambda)$ for some $\lambda \in \operatorname{Irr}(W)$. By Lemma 3.7 and Corollary 3.6. there is an action of $H_{c_{b}}\left(W_{b}, \mathfrak{h}_{b}\right)^{\wedge_{0}} \rtimes B_{N}$ on $\zeta\left(\operatorname{Res}_{b}(M)\right)^{\wedge_{0}}$. Since $(\cdot)^{\wedge_{0}}$ and $\zeta$ are equivalences of categories, we thus obtain an action of $H_{c_{b}}\left(W_{b}, \mathfrak{h}_{b}\right) \rtimes B_{N}$ on $\operatorname{Res}_{b}(M)$.

We use the argument from [10, §5.3] to extend this to any $M \in \mathcal{O}_{c}(W, \mathfrak{h})$. By 2.7 and 3.5, we can extend our constructions to the base rings $S$ and $K$ introduced in 2.6; there is an action of $H_{S}\left(W_{b}, \mathfrak{h}_{b}\right) \rtimes S B_{N}$ on $\operatorname{Res}_{b, S}\left(\Delta_{S}(\lambda)\right)$, and similarly for $\operatorname{Res}_{b, K}\left(\Delta_{K}(\lambda)\right)$. These actions are compatible with the natural maps $\mathbb{C} \nleftarrow S \hookrightarrow K$. Let $M \in \mathcal{O}_{S}^{\Delta}(W, \mathfrak{h})$. Since $M \otimes_{S} K$ embeds into a direct sum of standard modules, we establish the result for $M$ by using Lemma 2.7. By [10, Corollary 2.7] and basechange, the result holds for projective modules in $\mathcal{O}_{c}(W, \mathfrak{h})$. By construction, for any morphism between projective modules $P \rightarrow Q$, the resulting map $\operatorname{Res}_{b}(P) \rightarrow \operatorname{Res}_{b}(Q)$ is a map of $B_{N}$-modules. But for any $M \in \mathcal{O}_{c}(W, \mathfrak{h})$ there is an exact sequence $P_{1} \stackrel{f}{\rightarrow} P_{0} \rightarrow M \rightarrow 0$, where $P_{0}, P_{1} \in \mathcal{O}_{c}(W, \mathfrak{h})$ are projective. We deduce that $H_{c_{b}}\left(W_{b}, \mathfrak{h}_{b}\right) \rtimes B_{N}$ acts on $\operatorname{Res}_{b}(M) \cong \operatorname{Res}_{b}\left(P_{0}\right) / \operatorname{Im} \operatorname{Res}_{b}(f)$. The functoriality also follows.

3.9. We rephrase the statement of the theorem as the existence of an exact functor

$$
\operatorname{Res}_{W_{b}}^{W}: \mathcal{O}_{c}(W, \mathfrak{h}) \rightarrow\left(\mathcal{O}_{c_{b}}\left(W_{b}, \mathfrak{h}_{b}\right) \otimes \operatorname{Loc}\left(\mathfrak{h}_{\mathrm{r}}^{W_{b}}\right)\right)^{N_{W}\left(W_{b}\right)}
$$

where the superscript $N_{W}\left(W_{b}\right)$ denotes the $N_{W}\left(W_{b}\right)$-equivariant structure. This equivariant structure is obtained by extending the $N$-action to $N_{W}\left(W_{b}\right)=W_{b} \rtimes N$ by using the inner $W_{b}$ action on $H_{c_{b}}\left(W_{b}, \mathfrak{h}_{b}\right)$. The functor $\operatorname{Res}_{b}$ is the composition of $\operatorname{Res}_{W_{b}}^{W}$ with the functor that forgets the local system on $\mathfrak{h}_{\mathrm{r}}^{W_{b}}$ and the equivariant structure.

3.10. Decomposition of induction and restriction. This extra structure allows us to decompose both $\operatorname{Res}_{b}$ and $\operatorname{Ind}_{b}$. Consider again equivariant local systems on $\mathfrak{h}_{\mathrm{r}}^{W_{b}}$ as representations of $B_{N}$ and restrict them to $B_{C}$. Let $P \in \mathcal{O}(W, \mathfrak{h})$ be a projective generator, and let $A=$ $\mathbb{C}\left[B_{C}\right] / \operatorname{Ann}_{B_{C}}\left(\operatorname{Res}_{b}(P)\right)$, a finite dimensional algebra which is the image of $\mathbb{C}\left[B_{C}\right]$ in $\operatorname{End}_{\mathcal{O}_{c}(W, \mathfrak{h})}(P)$. Since $\operatorname{Res}_{b}$ is exact and $P$ is a generator, the $\mathbb{C}\left[B_{C}\right]$-action on $\operatorname{Res}_{b}(M)$ factors through $A$ for all 
$M \in \mathcal{O}_{c}(W, \mathfrak{h})$. Let $I$ denote a labelling set for the blocks of $A$ and for any $i \in I$ let $e_{i} \in Z(A)$ be the corresponding primitive central idempotent of $A$. By Theorem 3.8 we may consider $e_{i} \in \operatorname{End}\left(\operatorname{Res}_{b}\right)$ and then we have

$$
\operatorname{Res}_{b}(M)=\oplus_{i \in I} \operatorname{Res}_{b}^{i} \quad \text { where } \operatorname{Res}_{b}^{i}:=e_{i} \circ \operatorname{Res}_{b} .
$$

As $\left(\operatorname{Res}_{b}, \operatorname{Ind}_{b}\right)$ is an adjoint pair, we may apply [6, §4.1.5] to see that there exists corresponding adjoint pairs $\left(\operatorname{Res}_{b}^{i}, \operatorname{Ind}_{b}^{i}\right)$ for each $i \in I$ and such that $\operatorname{Ind}_{b}=\oplus_{i \in I} \operatorname{Ind}_{b}^{i}$.

3.11. Transitivity. Let us take two parabolic subgroups $W_{1} \leq W_{2}$. These produce decompositions $\mathfrak{h}=\mathfrak{h}^{W_{1}} \oplus \mathfrak{h}_{1}=\mathfrak{h}^{W_{2}} \oplus \mathfrak{h}_{2}$ and $\mathfrak{h}_{2}=\mathfrak{h}_{2}^{W_{1}} \oplus \mathfrak{h}_{1}$, where $\mathfrak{h}_{1}$ and $\mathfrak{h}_{2}$ are the non-trivial isotypic components of $\mathfrak{h}$ with respect to the actions of $W_{1}$ and $W_{2}$ respectively. Note that $\mathfrak{h}_{1} \subseteq \mathfrak{h}_{2}$ and $\mathfrak{h}^{W_{1}} \supseteq \mathfrak{h}^{W_{2}}$. By taking $W_{1}$-invariants we find $\mathfrak{h}^{W_{1}}=\mathfrak{h}^{W_{2}} \oplus \mathfrak{h}_{2}^{W_{1}}$. It is not true that one of $\mathfrak{h}_{\mathrm{r}}^{W_{1}}$ and $\mathfrak{h}_{\mathrm{r}}^{W_{2}} \times \mathfrak{h}_{2, \mathrm{r}}^{W_{1}}$ is contained in the other, but nevertheless we may pick $b_{1}, b_{2} \in \mathfrak{h}$ such that $b_{1} \in \mathfrak{h}_{\mathrm{r}}^{W_{1}}$ and $b_{2} \in \mathfrak{h}_{\mathrm{r}}^{W_{2}}$ and $b_{1}^{\prime}=\left(b_{2}^{\prime}, b_{1}^{\prime \prime}\right) \in \mathfrak{h}^{W_{2}} \times \mathfrak{h}_{2}^{W_{1}}$.

Let $N_{W}\left(W_{1}, W_{2}\right)=N_{W}\left(W_{1}\right) \cap N_{W}\left(W_{2}\right)$, which acts on $\mathfrak{h}_{\mathrm{r}}^{W_{1}}, \mathfrak{h}_{\mathrm{r}}^{W_{2}}$ and $\mathfrak{h}_{2, \mathrm{r}}^{W_{1}}$. There is a homomorphism

$$
\iota_{W_{1}, W_{2}}: \pi_{1}\left(\mathfrak{h}_{\mathrm{r}}^{W_{2}} \times \mathfrak{h}_{2, \mathrm{r}}^{W_{1}} / N_{W}\left(W_{1}, W_{2}\right),\left(b_{2}^{\prime}, b_{1}^{\prime \prime}\right)\right) \longrightarrow \pi_{1}\left(\mathfrak{h}_{\mathrm{r}}^{W_{1}} / N_{W}\left(W_{1}, W_{2}\right), b_{1}^{\prime}\right) .
$$

To construct this, note first that there is a free action of $N_{W}\left(W_{1}, W_{2}\right) / W_{1}$ on both $\mathfrak{h}_{\mathrm{r}}^{W_{2}} \times \mathfrak{h}_{2, \mathrm{r}}^{W_{1}}$ and $\mathfrak{h}_{\mathrm{r}}^{W_{1}}$. Therefore it is enough to produce an $N_{W}\left(W_{1}, W_{2}\right)$-equivariant homomorphism from homotopy classes of paths in $\mathfrak{h}_{\mathrm{r}}^{W_{2}} \times \mathfrak{h}_{2, \mathrm{r}}^{W_{1}}$ to homotopy classes of paths in $\mathfrak{h}_{\mathrm{r}}^{W_{1}}$. Given a path $\gamma=\left(\gamma_{1}, \gamma_{2}\right):[0,1] \rightarrow \mathfrak{h}_{\mathrm{r}}^{W_{2}} \times \mathfrak{h}_{2, \mathrm{r}}^{W_{1}}$, we may adjust $\gamma_{2}$ sufficiently inside $\mathfrak{h}_{2, \mathrm{r}}^{W_{1}}$, depending on how close $\gamma_{1}$ passes to the reflecting hyperplanes in $\mathfrak{h}$ that do not contain $\mathfrak{h}^{W_{2}}$, to ensure that the image of $\gamma$ belongs to $\mathfrak{h}_{\mathrm{r}}^{W_{1}}$. Inclusion then provides the homomorphism.

Theorem. There is a natural isomorphism of functors from $\mathcal{O}(W, \mathfrak{h})$ to $\left(\mathcal{O}\left(W_{1}, \mathfrak{h}_{1}\right) \otimes \operatorname{Loc}\left(\mathfrak{h}_{2, r}^{W_{1}}\right) \otimes\right.$ $\left.\operatorname{Loc}\left(\mathfrak{h}_{r}^{W_{2}}\right)\right)^{N_{W}\left(W_{1}, W_{2}\right)}$,

$$
\iota_{W_{1}, W_{2}}^{*} \circ \downarrow_{N_{W}\left(W_{1}, W_{2}\right)}^{N_{W}\left(W_{1}\right)} \circ \operatorname{Res}_{W_{1}}^{W} \cong \operatorname{Res}_{W_{1}}^{W_{2}} \otimes \operatorname{id} \circ \downarrow_{N_{W}\left(W_{1}, W_{2}\right)}^{N_{W}\left(W_{2}\right)} \circ \operatorname{Res}_{W_{2}}^{W},
$$

where $\downarrow$ denotes the restriction of equivariant structure to a subgroup.

Proof. It is unpleasant to deal directly with the path manipulation appearing in the construction of $\iota_{W_{1}, W_{2}}$. To avoid this we work instead with an intermediate version of Theorems 2.2 and 2.9 and the restriction functors. For $b \in \mathfrak{h}$ with stabilizer $W_{b}$ this version takes place within a formal neighbourhood of $W \cdot \mathfrak{h}_{\mathrm{r}}^{W_{b}}$ in $\mathfrak{h}$. The space $\mathfrak{h}_{\mathrm{r}}^{W_{b}}$ is an affine open algebraic subset of $\mathfrak{h}^{W_{b}}$ given by the non-vanishing of the polynomial $\pi:=\prod_{s \in \mathcal{S} \backslash W_{b}} \alpha_{s}$. Set $\bar{\pi}=\prod_{w \in W} w \cdot \pi$, a polynomial whose non-vanishing defines $W \cdot \mathfrak{h}_{\mathrm{r}}^{W_{b}}=\left\{x \in \mathfrak{h} \mid W_{x}\right.$ is conjugate to $\left.W_{b}\right\}$. We denote by $H_{c}(W, \mathfrak{h})^{\wedge}{ }^{\left[\mathfrak{h}_{\mathrm{r}}{ }^{W_{b}}\right]}$ the subalgebra of $\mathbb{C}$-linear endomorphisms of $\mathbb{C}[\mathfrak{h}]^{\wedge} \hat{\mathfrak{h}}_{\mathrm{r}}^{\left.W_{b}\right]}:=\mathbb{C}[\mathfrak{h}]^{\wedge}\left[\mathfrak{h}^{\left.W_{b}\right]}\left[\bar{\pi}^{-1}\right]\right.$ generated by $\mathbb{C}[\mathfrak{h}]^{\wedge}\left[\mathfrak{h r}^{\left.W_{b}\right]}, D_{y}\right.$ for $y \in \mathfrak{h}$ and $w \in W$. There is then an isomorphism of algebras

$$
\Theta_{b}: H_{c}(W, \mathfrak{h})^{\wedge}\left[\mathfrak{h}_{\mathrm{r}}^{\left.W_{b}\right]} \longrightarrow Z\left(W, W_{b}, H_{c_{b}}\left(W_{b}, \mathfrak{h}\right) \hat{\wedge}_{\mathfrak{h}_{\mathrm{r}}}^{W_{b}}\right),\right.
$$

which is defined exactly as in Theorem 2.2. There is furthermore an isomorphism $H_{c_{b}}\left(W_{b}, \mathfrak{h}\right) \wedge^{\wedge} W_{\mathrm{r}} W_{b} \cong$ $\mathcal{D}_{\text {pol }}\left(\mathfrak{h}_{\mathrm{r}}^{W_{b}}\right) \otimes H_{c_{b}}\left(W_{b}, \mathfrak{h}_{b}\right)^{\wedge_{0}}$. The restriction functors are then defined as usual, splitting the centralizer with an element we label by $1_{W_{b}}^{W}$, then taking locally-finite vectors with respect to eu $\in H_{c_{b}}\left(W_{b}, \mathfrak{h}\right)$. 
Now we move onto the proof. Corresponding to the natural algebra homomorphisms

$$
\begin{aligned}
\mathbb{C}[\mathfrak{h}]^{\wedge} \mathfrak{h}_{\mathrm{r}}^{W_{1}}=\mathbb{C}\left[\mathfrak{h}_{\mathrm{r}}^{W_{1}}\right] \otimes \mathbb{C}\left[\left[\mathfrak{h}_{1}\right]\right] & \rightarrow \mathbb{C}\left[\mathfrak{h}_{\mathrm{r}}^{W_{2}}\right] \otimes \mathbb{C}\left[\left[\mathfrak{h}_{2}^{W_{1}}\right]\right]\left[\alpha_{s}^{-1}: s \in W_{2} \backslash W_{1}\right] \otimes \mathbb{C}\left[\left[\mathfrak{h}_{1}\right]\right] \\
& \rightarrow \mathbb{C}\left[\mathfrak{h}_{\mathrm{r}}^{W_{2}}\right] \otimes \mathbb{C}\left[\left[\mathfrak{h}_{2}^{W_{1}} \times \mathfrak{h}_{1}\right]\right]\left[\alpha_{s}^{-1}: s \in W_{2} \backslash W_{1}\right]=\left.\mathbb{C}[\mathfrak{h}]^{\wedge} \mathfrak{h}_{\mathrm{r}}^{W_{2}}\right|_{\mathfrak{h}_{2, \mathrm{r}}^{W_{1}} \times \mathfrak{h}_{1}}
\end{aligned}
$$

we see that successive restriction to smaller formal neighbourhoods produces $1_{W_{2}}^{W}\left(M^{\wedge}\left[\mathrm{lb}_{\mathrm{r}}^{W_{1}}\right]\right) \mapsto$ $\left.1_{W_{2}}^{W}\left(M^{\left.{ }^{\left[\mathfrak{h}_{\mathrm{r}}\right.} W_{2}\right]}\right)\right|_{W_{2} \cdot\left(\mathfrak{h}_{2, \mathrm{r}}^{W_{1}} \times \mathfrak{h}_{1}\right)}$. The space $1_{W_{1}}^{W}\left(M^{\wedge} \hat{\mathfrak{l}}_{\mathrm{r}}^{\left.W_{1}\right]}\right)$ has a natural $D\left(\mathfrak{h}_{\mathrm{r}}^{W_{1}}\right)$-structure which extends to a $\mathcal{D}\left(\mathfrak{h}_{\mathrm{r}}^{W_{2}}\right) \otimes \mathcal{D}\left(\mathfrak{h}_{2, \mathrm{r}}^{W_{1}}\right)^{\wedge} 0$-structure on $1_{W_{1}}^{W_{2}}\left(\left.1_{W_{2}}^{W_{2}}\left(M^{\wedge\left[\mathfrak{h}_{\mathrm{r}}^{W_{2}}\right]}\right)\right|_{W_{2} \cdot\left(\mathfrak{h}_{2, \mathrm{r}}^{W_{1}} \times \mathfrak{h}_{1}\right)}\right)$.

The natural inclusion

$$
\left(\left.\left(1_{W_{2}}^{W}\left(M^{\wedge}\left[\mathfrak{h}_{\mathrm{r}}^{\left.W_{2}\right]}\right)\right)^{\mathbf{e u}}\right)\right|_{W_{2} \cdot\left(\mathfrak{h}_{2, \mathrm{r}}^{W_{1}} \times \mathfrak{h}_{1}\right)} \rightarrow\left(\left.\left(1_{W_{2}}^{W}\left(M^{\wedge\left[\mathfrak{h}_{\mathrm{r}}^{W_{2}}\right]}\right)\right)\right|_{W_{2} \cdot\left(\mathfrak{h}_{2, \mathrm{r}}^{W_{1}} \times \mathfrak{h}_{1}\right)}\right)^{\mathbf{e u}}\right.
$$

is an isomorphism since the $\left\{\alpha_{s}\right\}_{s \in W_{2}}$ have positive $\mathbf{e u}_{2}$-weights. For $y \in \mathfrak{h}_{\mathrm{r}}^{W_{1}} \subset \mathfrak{h}_{2}$ we have that

$$
\partial_{y}=D_{y}-\sum_{s \in W_{2} \backslash W_{1}} \frac{2 c_{s}}{1-\operatorname{det}_{\mathfrak{h}^{*}}(s)} \frac{\alpha_{s}(y)}{\alpha_{s}}(s-1) \in H_{c_{2}}\left(W_{2}, \mathfrak{h}_{2}\right)\left[\alpha_{s}^{-1}: s \in W_{2} \backslash W_{1}\right]
$$

so that there is an action of $D\left(\mathfrak{h}_{\mathrm{r}}^{W_{2}}\right) \otimes D\left(\mathfrak{h}_{2, \mathrm{r}}^{W_{1}}\right)$ on $\left(\left.\left(1_{W_{2}}^{W}\left(M^{\wedge}{ }^{\left[\mathfrak{h}_{\mathrm{r}}{ }^{W_{2}}\right]}\right)\right)\right|_{W_{2} \cdot\left(\mathfrak{h}_{2, \mathrm{r}}^{W_{1}} \times \mathfrak{h}_{1}\right)}\right)^{\text {eu }}{ }^{\mathbf{u}_{2}}$. Completing at $0 \in \mathfrak{h}_{2}^{W_{1}}$ produces another action of $\mathcal{D}\left(\mathfrak{h}_{\mathrm{r}}^{W_{2}}\right) \otimes \mathcal{D}\left(\mathfrak{h}_{2, \mathrm{r}}^{W_{1}}\right)^{\wedge} 0$ on $\left.\left(1_{W_{2}}^{W}\left(M^{\wedge}\left[\mathfrak{h}_{\mathrm{r}}^{W_{2}}\right]\right)\right)\right|_{W_{2} \cdot\left(\mathfrak{h}_{2, \mathrm{r}}^{W_{1}} \times \mathfrak{h}_{1}\right)}$.

A similar argument to [18, Lemma 2.2], using the version of the comparison theorem over the formal neighbourhood of a subvariety due to Kashiwara-Schapira, [12, Corollary 6.2], allows us to deduce that the monodromy representations of the two above local systems on $\mathfrak{h}_{\mathrm{r}}^{W_{2}} \times \mathfrak{h}_{2, r}^{W_{1}}$ agree.

We then have a functorial morphism

$$
\begin{aligned}
& 1_{W_{1}}^{W}\left(M^{\wedge}{ }^{\left[\mathfrak{h}_{\mathrm{r}}^{W_{1}}\right]}\right)^{\mathbf{e u}} \mapsto 1_{W_{1}}^{W_{2}}\left(\left.1_{W_{2}}^{W}\left(M^{\left[\mathfrak{h}_{\mathrm{r}}^{W_{2}}\right]}\right)\right|_{W_{2} \cdot\left(\mathfrak{h}_{2, \mathrm{r}}^{W_{1}} \times \mathfrak{h}_{1}\right)}\right)^{\mathbf{e u}} \quad \mapsto 1_{W_{1}}^{W_{2}}\left(\left(1_{W_{2}}^{W}\left(\left.M^{\wedge}\left[\mathfrak{l}_{\mathrm{r}}^{\left.W_{2}\right]}\right)\right|_{W_{2} \cdot\left(\mathfrak{h}_{2, \mathrm{r}}^{W_{1}} \times \mathfrak{h}_{1}\right)}\right)^{\mathbf{e u _ { 2 }}}\right)^{\mathbf{e u _ { 1 }}}\right. \\
& \mapsto 1_{W_{1}}^{W_{2}}\left(\left.\left(1_{W_{2}}^{W}\left(M^{\wedge}{ }_{\left[\mathfrak{h}_{\mathrm{r}}^{W_{2}}\right]}\right)^{\mathbf{e u}}\right)\right|_{W_{2} \cdot\left(\mathfrak{h}_{2, \mathrm{r}}^{W_{1}} \times \mathfrak{h}_{1}\right)}\right)^{\mathbf{e u}} \\
& \mapsto 1_{W_{1}}^{W_{2}}\left(\left(1_{W_{2}}^{W}\left(M^{\wedge} \hat{[h r}_{\mathrm{r}}^{\left.W_{2}\right]}\right)^{\mathbf{e u}_{2}}\right)^{\wedge} \mathfrak{[ h}_{2, \mathrm{r}}^{\left.W_{1}\right]}\right) \mathbf{e u}_{1}
\end{aligned}
$$

This realizes the functor $\iota_{W_{1}, W_{2}}^{*}$. Since the initial term is $\operatorname{Res}_{W_{1}}^{W}(M)$ and the final term is $\operatorname{Res}_{W_{1}}^{W_{2}}\left(\operatorname{Res}_{W_{2}}^{W}(M)\right)$, this completes the proof.

\section{Consequences for $G(\ell, 1, n)$}

4.1. The groups $G(\ell, 1, n)$. Fix $\ell \in \mathbb{N}$, and let $W_{n}=W(G(\ell, 1, n))=\mu_{\ell}^{n} \rtimes \mathfrak{S}_{n}$ for any $n \in \mathbb{N}$, a complex reflection group with a Coxeter style presentation

$$
\left.\left\langle t, s_{1}, \ldots, s_{n-1}\right| s_{i}^{2}=t^{\ell}=1, s_{i} s_{i+1} s_{i}=s_{i+1} s_{i} s_{i+1}, s_{i} s_{j}=s_{j} s_{i} \text { if }|i-j|>1, s_{1} t s_{1} t=t s_{1} t s_{1}\right\rangle .
$$

The reflection representation $\mathfrak{h}_{n}=\mathfrak{h}$ of $W_{n}$ is the vector space $\mathbb{C}^{n}=\operatorname{span}\left\{y_{1}, \ldots, y_{n}\right\}$. With respect to the standard basis the $s_{i}$ generate a copy of the symmetric group acting by place permutation, and $t$ acts by $\operatorname{diag}(\eta, 1, \ldots, 1)$, where $\eta=\exp (2 \pi \sqrt{-1} / \ell)$. We write $t_{i}=(1, \ldots, 1, \eta, 1, \ldots, 1)$ where $\eta$ appears at the $i^{\text {th }}$ coordinate. 
4.2. When $n>1$ there are $\ell$ conjugacy classes of reflections, the set of conjugates of the $s_{i}$ and the set of conjugates of $t^{r}$ for $1 \leq r \leq \ell-1$; when $n=1$ there are no $s_{i}$ and so only $\ell-1$ classes. The parameters we choose for the rational Cherednik algebra are

$$
c_{s_{i}}=-k \text { and } c_{t^{r}}=-\frac{1}{2}\left(1+\sum_{j=1}^{\ell} k\left(m_{j+1}-m_{j}\right) \eta^{-r j}\right) \text { for } 1 \leq r \leq \ell-1
$$

where $k \in \mathbb{C}, \mathbf{m}=\left(m_{1}, \ldots, m_{\ell}\right) \in \mathbb{Z}^{\ell}$ and we set $m_{\ell+1}=m_{1}$.

4.3. We identify the irreducible representations $\operatorname{Irr}\left(W_{n}\right)$ of $W_{n}$ with the set $\mathcal{P}_{\ell}(n)$ of $\ell$-multipartitions of $n$, [17, 6.1.1]. Set $\mathcal{P}_{\ell}=\bigcup_{n \geq 0} \mathcal{P}_{\ell}(n)$. We write $\lambda=\left(\lambda^{1}, \ldots, \lambda^{\ell}\right)$ for the multipartition and the corresponding representation and we will often identify $\lambda$ with an $\ell$-tuple of Young diagrams. If a box $p \in \lambda$ is in position $(i, j)$ of the Young diagram of $\lambda^{t}$ we set $\beta(p)=t$, and define the residue as $\operatorname{res}(p)=j-i$ and the $\mathbf{m}$-shifted residue $\operatorname{res}^{\mathbf{m}}(p)=\operatorname{res}(p)+m_{\beta(p)}$.

4.4. Induction and restriction. Set $b_{n}=b=(0, \ldots, 0, n)$. Then $W_{b}=W_{n-1}, N_{W}\left(W_{n-1}\right)=$ $W_{n-1} \times N$ where $N=\left\langle t_{n}\right\rangle \cong \mu_{\ell}$. Clearly $\mathfrak{h}_{b}=\left\{\left(a_{1}, a_{2}, \ldots, a_{n-1}, 0\right): a_{i} \in \mathbb{C}\right\}$ and $\mathfrak{h}_{\mathrm{r}}^{W_{n-1}}=$ $\left\{\left(0, \ldots, 0, a_{n}\right): 0 \neq a_{n} \in \mathbb{C}\right\}$. We have

$$
\operatorname{Res}_{W_{n-1}}^{W_{n}}: \mathcal{O}_{c}\left(W_{n}\right) \rightarrow\left(\mathcal{O}_{c}\left(W_{n-1}\right) \otimes \operatorname{Loc}\left(\mathfrak{h}_{\mathrm{r}}^{W_{n-1}}\right)\right)^{N_{W}\left(W_{n-1}\right)}
$$

Let $\lambda \in \operatorname{Irr}(W)$. Recall from Proposition 3.5 that the local system on $\mathfrak{h}_{\mathrm{r}}^{W_{n-1}} \cong \mathbb{C}^{\times}$attached to $\operatorname{Res}_{W_{n-1}}^{W}(\Delta(\lambda))$ arises from the $N$-equivariant connection

$$
\sum_{s \in \mathcal{S} \backslash W_{n-1}} \frac{2 c_{s}}{1-\operatorname{det}_{\mathfrak{h}^{*}}(s)} \frac{d \alpha_{s}}{\alpha_{s}}(s-1)
$$

on $\operatorname{Hom}_{W_{n-1}}\left(\nu, \lambda \downarrow_{W_{n-1}}\right)$ where we run over all $\nu \in \operatorname{Irr}\left(W_{n-1}\right)$.

There are two types of $s \in \mathcal{S} \backslash W_{n-1}$ : $(i n) t_{i}^{r} t_{n}^{-r}$ for $1 \leq i \leq n-1,0 \leq r \leq \ell-1$, with $\alpha_{s}=-\eta^{r} y_{i}+y_{n} ; t_{n}^{r}$ for $1 \leq r \leq \ell-1$ with $\alpha_{s}=y_{n}$. Thus the above connection can be written explicitly as the following $\mu_{\ell}$-equivariant connection on $\bigoplus_{\nu \in \operatorname{Irr}\left(W_{n-1}\right)} \operatorname{Hom}_{W_{n-1}}\left(\nu, \lambda \downarrow_{W_{n-1}}\right)$ over $\mathbb{C}^{\times}$

$$
-\sum_{i=1}^{n-1} \sum_{r=0}^{\ell-1} k \frac{d z}{z}\left((i n) t_{i}^{r} t_{n}^{-r}-1\right)-\sum_{r=1}^{\ell-1} \frac{1+\sum_{j=1}^{\ell} k\left(m_{j+1}-m_{j}\right) \eta^{-r j}}{1-\eta^{-r}} \frac{d z}{z}\left(t_{n}^{r}-1\right) .
$$

4.5. To calculate the monodromy of this connection we will apply the following result which essentially appears in [4, Theorem 4.12]

Lemma. Let $\mathcal{V}$ be a trivial vector bundle on $\mathbb{C}^{\times}$, equipped with $\mu_{\ell}$-equivariant structure and take a $\mu_{\ell}$-equivariant connection on $\mathcal{V}$ of the form $\omega=\sum_{r=0}^{\ell-1} \alpha_{r} \epsilon_{r} \frac{d z}{z}$, where $\epsilon_{r}=\frac{1}{\ell} \sum_{j=0}^{l-1} \exp (-2 \pi \sqrt{-1} r)^{j} t_{n}^{j} \in$ $\mathbb{C}\left[\mu_{\ell}\right]$. Let $\Sigma$ be the monodromy operator corresponding to the generator of $\pi_{1}\left(\mathbb{C}^{\times}, \cdot\right)$, an anticlockwise loop about the origin. Then the part of the $\Sigma$ corresponding to the term $\alpha_{r} \epsilon_{r} d z / z$ is given by multiplication by $\exp \left(2 \pi \sqrt{-1}\left(\alpha_{r}-r\right) / \ell\right)$. 
4.6. The space $M=\operatorname{Hom}_{W_{n-1}}\left(\nu, \lambda \downarrow_{W_{n-1}}\right)$ is either zero or one-dimensional, with the non-zero spaces occuring precisely when $p$ is a box of $\lambda$ such that $\nu=\lambda \backslash\{p\}$. Assume we are in this case. Then $t_{n}$ acts on $M$ by multiplication by $\eta^{\beta(p)-1}$. The element $\sum_{i=1}^{n-1} \sum_{r=0}^{\ell-1}(i n) t_{i}^{r} t_{n}^{-r}$ is the $n$th Jucys-Murphy element of $\mathbb{C}\left[W_{n}\right]$ : it acts on $M$ by multiplication by $\ell \cdot \operatorname{res}(p)$. Therefore on $M$ the coefficient of the connection is multiplication by $k \ell(n-1)-k \ell \operatorname{res}(p)+k \ell\left(m_{1}-m_{\beta(p)}\right)+\beta(p)-1$. Thus by Lemma 4.5 the monodromy $\Sigma_{\Delta(\lambda)}$ of the local system arising from $\operatorname{Res}_{W_{n-1}}^{W_{n}}(\Delta(\lambda))$ satisfies the relation

$$
\prod_{p}\left(\Sigma_{\Delta(\lambda)}-q^{\left(\operatorname{res}^{\mathbf{m}}(p)+1-n\right)-m_{1}}\right)=0,
$$

where $p$ runs over all removable boxes of $\lambda$ and $q=\exp (-2 \pi \sqrt{-1} k)$.

Proposition. Let $M \in \mathcal{O}_{c}\left(W_{n}\right)$ and let $\Sigma_{M}$ denote the monodromy operator on $\operatorname{Res}_{b}(M)$ arising from the local system over $\mathfrak{h}_{r}^{W_{n-1}}$ attached to $\operatorname{Res}_{W_{n-1}}^{W_{n}}(M)$. The eigenvalues of $\Sigma_{M}$ lie in $\left\{q^{i} \mid i \in \mathbb{Z}\right\}$.

Proof. By the calculation above we know that $\Sigma_{\Delta(\lambda)}$ has eigenvalues in this set for all $\lambda \in \operatorname{Irr}\left(W_{n}\right)$. Since $\operatorname{Res}_{b}(L(\lambda))$ is a quotient of $\operatorname{Res}_{b}(\Delta(\lambda))$, the same is true for any $\Sigma_{L(\lambda)}$ and so for any $\Sigma_{M}$.

4.7. We can now decompose $\operatorname{Res}_{b}$ as in 3.10, Let $i$ be an integer. We define the functor $\operatorname{Res}^{i}(n):=$ $\pi_{i} \circ \operatorname{Res}_{b}$, where $\pi_{i}$ is projection on to the $q^{i+1-n-m_{1}}$ generalized eigenspace of the monodromy operator $\Sigma$. We have a decomposition

$$
\operatorname{Res}_{b}=\bigoplus_{i \in \mathbb{Z} / \sim} \operatorname{Res}^{i}(n)
$$

where $i \sim j$ if and only $q^{i}=q^{j}$.

Let $\operatorname{Ind}^{i}(n)$ denote the right adjoint of $\operatorname{Res}^{i}(n)$.

4.8. $\tilde{\mathfrak{s l}}_{e}$-categorification. We proceed to the theorem which has been proved by Shan in [18, Theorem 5.1] using the KZ-functor and its double centralizer property. We will show in 4.13 our approach is identical to [18]. Nonetheless, we outline the result and its proof here as it avoids using the double centralizer.

4.9. Recall the choice of parameters from 4.2: $k \in \mathbb{C}$ and $\mathbf{m}=\left(m_{1}, \ldots, m_{\ell}\right) \in \mathbb{Z}^{\ell}$. Let $e^{\prime} \in \mathbb{N} \cup\{\infty\}$ be the multiplicative order of $q=\exp (-2 \pi \sqrt{-1} k) \in \mathbb{C}^{\times}$and set $e=e^{\prime}$ if $e^{\prime} \neq 1$ and set $e=\infty$ if $e^{\prime}=1$. Let $\mathcal{F}(\mathbf{m})$ denote the Fock space with multicharge $\mathbf{m}$, an integrable $\tilde{\mathfrak{s l}}_{e}$-representation, see for instance [11]. As a vector space we have $\mathcal{F}(\mathbf{m})=\bigoplus_{\lambda \in \mathcal{P}_{\ell}} \mathbb{C} \lambda$; for $i \in \mathbb{Z} / e \mathbb{Z}$ the corresponding Chevalley generators act as

$$
e_{i}(\lambda)=\sum_{|\lambda / \mu|=1, \operatorname{res}^{\mathbf{m}}(\lambda / \mu) \equiv i} \mu, \quad f_{i}(\lambda)=\sum_{|\mu / \lambda|=1, \mathrm{res}^{\mathbf{m}}(\mu / \lambda) \equiv i} \mu
$$

finally $\partial(\lambda)=-\tau_{0} \lambda$ where $\tau_{0}$ is the number of boxes in $\lambda$ with $\mathbf{m}$-shifted residue divisible by $e$. The weight spaces of $\mathcal{F}(m)$ are $\mathcal{F}(m)_{\tau}$ for $\tau=\left(\tau_{0}, \ldots, \tau_{e-1}\right) \in \mathbb{Z}_{\geq 0} \times \mathbb{Z} / e \mathbb{Z}$ where $\mathcal{F}(m)_{\tau}$ is spanned by the multipartitions having exactly $\tau_{i}$ boxes with $\mathbf{m}$-shifted residue equal to $i$ for each $i \in \mathbb{Z} / e \mathbb{Z}$. Such elements have weight $\sum_{j=1}^{\ell} \Lambda_{m_{j}}-\sum_{i \in \mathbb{Z} / e \mathbb{Z}} \tau_{i} \alpha_{i}$ where $\Lambda_{i}$ is the $i^{\text {th }}$ fundamental weight of $\tilde{\mathfrak{s}}_{e}$. 
4.10. Let $\mathcal{O}_{c}(\mathbb{N})=\bigoplus_{n \geq 0} \mathcal{O}_{c}\left(W_{n}, \mathfrak{h}_{n}\right)$. Set $E=\bigoplus_{n \geq 0} \operatorname{Res}_{b_{n}}, F=\bigoplus_{n \geq 0} \operatorname{Ind}_{b_{n}}: \mathcal{O}_{c}(\mathbb{N}) \rightarrow \mathcal{O}_{c}(\mathbb{N})$. Define $X \in \operatorname{End}(E)$ as the direct sum over $n \geq 0$ of the operators $\Sigma \in \pi_{1}\left(\mathfrak{h}_{\mathrm{r}}^{W_{n-1}} / \mu_{\ell}, \cdot\right)$. By Theorem 3.11 we may identify $E^{2}$ with the direct sum of the restrictions $\operatorname{Res}_{\tilde{b}_{n}}: \mathcal{O}\left(W_{n}, \mathfrak{h}_{n}\right) \rightarrow$ $\mathcal{O}\left(W_{n-2}, \mathfrak{h}_{n-2}\right)$ where $\tilde{b}_{n}=(0, \ldots, 0, n-1, n) \in \mathfrak{h}_{n}$. Then $\mathfrak{h}_{\mathrm{r}}^{W_{\tilde{b}_{n}}}=\left\{\left(a_{n-1}, a_{n}\right) \in \mathbb{C}^{2}: a_{n-1}, a_{n} \neq\right.$ 0 and $a_{n-1} \neq \eta^{j} a_{n}$ for $\left.0 \leq j \leq \ell-1\right\}$ and $N\left(W_{\tilde{b}_{n}}\right)=W_{\tilde{b}_{n}} \rtimes W_{2}^{\prime}$ where $W_{2}^{\prime}=\left\langle s_{n-1}, t_{n-1}, t_{n}\right\rangle$. Let $T \in \operatorname{End}\left(\operatorname{Res}_{\tilde{b}_{n}}\right)$ be the operator arising from the generator of monodromy in $\pi_{1}\left(\mathfrak{h}_{\mathrm{r}} W_{\tilde{b}_{n}} / W_{2}^{\prime}, \cdot\right)$ attached to the reflecting hyperplane $a_{n-1}=a_{n}$ in $\mathfrak{h}^{W_{\tilde{b}_{n}}}$, [4, Appendix 1]. We may decompose $\mathcal{O}_{c}(\mathbb{N})=\bigoplus_{\tau} \mathcal{O}_{c}(\mathbb{N})_{\tau}$ where $\tau=\left(\tau_{0}, \ldots, \tau_{e-1}\right)$ and $\mathcal{O}_{c}(\mathbb{N})_{\tau}$ is the full subcategory of $\mathcal{O}_{c}\left(W_{|\tau|}, \mathfrak{h}_{|\tau|}\right)$ generated by all the $L(\lambda)$ where $\lambda$ has exactly $\tau_{i}$ boxes with $\mathbf{m}$-shifted residue equal to $i$ for each $i \in \mathbb{Z} / e \mathbb{Z}$. By [7, Theorem 4.1] $\mathcal{O}_{c}(\mathbb{N})_{\tau}$ is a sum of blocks of $\mathcal{O}_{c}(\mathbb{N})$.

Theorem. Let $c$ be the parameters for the rational Cherednik algebra given in 4.2 and keep the notation above.

(1) The adjoint pair $(E, F), X \in \operatorname{End}(E), T \in \operatorname{End}\left(E^{2}\right)$ and the block decomposition $\mathcal{O}_{c}(\mathbb{N})=$ $\bigoplus_{\tau \in P} \mathcal{O}_{c}(\mathbb{N})_{\tau}$ gives an $\tilde{\mathfrak{s l}}_{e}$-categorification of $\mathcal{F}(\mathbf{m})$ by $\mathcal{O}_{c}(\mathbb{N})$, .

(2) The simple objects in $\mathcal{O}_{c}(\mathbb{N})$ give an $\tilde{\mathfrak{s l}}_{e}$-crystal basis for the Grothendieck group $\left[\mathcal{O}_{c}(\mathbb{N})\right]$ which is isomorphic to the crystal of the Fock space $\mathcal{F}(\mathbf{m})$.

Proof. (1) By construction the eigenoperators of $E$ under the action of $X$ are the sums of $\operatorname{Res}^{i}(n)$ over all $n$. The standard modules $\left\{[\Delta(\lambda)]: \lambda \in \mathcal{P}_{\ell}\right\}$ give a basis of $\mathbb{Q} \otimes K\left(\mathcal{O}_{c}(\mathbb{N})\right)$. Identify $\mathbb{Q} \otimes K\left(\mathcal{O}_{c}(\mathbb{N})\right)$ with $\mathcal{F}(\mathbf{m})$ by sending $[\Delta(\lambda)]$ to $\lambda$. We have seen in 4.6 that $\operatorname{Res}^{i}([\Delta(\lambda)])=$ $\sum_{|\lambda / \mu|=1, \operatorname{res}^{\mathbf{m}}(\lambda / \mu) \equiv i}[\Delta(\mu)]$ for any $\lambda \in \mathcal{P}_{\ell}$. It then follows by adjunction and the elementary fact that $\operatorname{Res}^{i}([\Delta(\lambda)])$ has a standard filtration, see [18, Proposition 1.9], that $\operatorname{Ind}^{i}([\nabla(\lambda)])=$ $\sum_{|\mu / \lambda|=1, \operatorname{res}^{\mathbf{m}}(\mu / \lambda) \equiv i}[\nabla(\mu)]$ where $\nabla(-)$ denotes a costandard module in $\mathcal{O}_{c}(\mathbb{N})$. Since $[\Delta(\lambda)]=$ $[\nabla(\lambda)]$ for any $\lambda \in \mathcal{P}_{\ell},\left[10\right.$, Proposition 3.3], it follows that we have a weak $\tilde{\mathfrak{s l}}_{e}$-categorification of $\mathcal{F}(\mathbf{m})$.

For the full $\tilde{\mathfrak{s l}}_{e}$-categorification we also need that $F$ is a left adjoint of $E$ : this is a theorem of Shan, [18, Proposition 2.9], and Losev, 13]. Finally, the compatibilities and equalities required of $T$ and $X$ all follow from Theorem 3.11 and standard monodromy calculations in $\mathfrak{h}_{\mathrm{r}}^{W_{\tilde{b}_{n}}}$ and $\mathfrak{h}_{\mathrm{r}}^{W_{\hat{b}_{n}}}$ with the connections of Proposition 3.5. where $\tilde{b}_{n}=(0, \ldots, 0, n-1, n)$ and $\hat{b}_{n}=(0, \ldots, n-2, n-1, n)$.

(2) This follows formally, as explained in [18, Theorem 6.3].

4.11. Monodromy and the $\mathbf{K Z}$ functor. We now compare $\operatorname{Res}^{i}(n)$ with $E_{i}(n)$, the functor of $i$-restriction from [18, Definition 4.2]. We choose the basepoint $x_{0}=(1,2, \ldots, n) \in \mathfrak{h}_{\mathrm{r}}^{\{1\}}=: \mathfrak{h}_{\mathrm{r}}$ and recall that the KZ-functor $\mathrm{KZ}_{n}: \mathcal{O}_{c}\left(W_{n}, \mathfrak{h}\right) \rightarrow \mathcal{H}_{q}\left(W_{n}\right)$-mod is obtained from the local system over $\mathfrak{h}_{\mathrm{r}} / W_{n}$ attached to $\operatorname{Res}_{\{1\}}^{W_{n}}$. We have $\pi_{1}\left(\mathfrak{h}_{\mathrm{r}} / W_{n}, x_{0}\right)=B_{n}$, the braid group attached to $W_{n}$, which following [4] may be presented as

$$
\left.\left\langle\tau, \sigma_{1}, \ldots, \sigma_{n-1}\right| \sigma_{i} \sigma_{i+1} \sigma_{i}=\sigma_{i+1} \sigma_{i} \sigma_{i+1}, \sigma_{i} \sigma_{j}=\sigma_{j} \sigma_{i} \text { if }|i-j|>1, \sigma_{1} \tau \sigma_{1} \tau=\tau \sigma_{1} \tau \sigma_{1}\right\rangle,
$$

and $\mathcal{H}_{q}\left(W_{n}\right)$ is the quotient of $\mathbb{C}\left[B_{n}\right]$ by the relations

$$
\left(\sigma_{i}-1\right)\left(\sigma_{i}+q^{-1}\right)=0 \text { for } 1 \leq i \leq n-1, \text { and } \prod_{i=1}^{\ell}\left(\tau-q^{m_{i}-m_{1}}\right)=0
$$


4.12. There is an algebra isomorphism $\gamma: Z\left(\mathcal{O}_{c}\left(W_{n}, \mathfrak{h}\right)\right) \stackrel{\sim}{\rightarrow} Z\left(\mathcal{H}_{q}\left(W_{n}\right)\right)$ such that $z M=\gamma(z) \mathrm{KZ}_{n}(M)$ for all $z \in Z\left(\mathcal{O}_{c}\left(W_{n}, \mathfrak{h}\right)\right)$. Let $J_{0}, \ldots, J_{n-1}$ denote the Jucys-Murphy elements in $\mathcal{H}_{q}\left(W_{n}\right)$, defined by

$$
\mathrm{J}_{0}=q^{m_{1}} \tau, \mathrm{J}_{i}=q^{m_{1}+i-1} \sigma_{i} \cdots \sigma_{1} \tau \sigma_{1} \ldots \sigma_{i},
$$

for $1 \leq i \leq n-1$. (See [9, Definition 5.2.3], but note that our normalization of the Hecke algebra $\mathcal{H}_{q}\left(W_{n}\right)$ differs from [loc.cit] so we have a slightly different definition.) Set $C_{n}(z)=\prod_{i=0}^{n-1}\left(z-J_{i}\right)$, a polynomial in the variable $z$ whose coefficients lie in $Z\left(\mathcal{H}_{q}\left(W_{n}\right)\right)$. Let $D_{n}(z)=\gamma^{-1}\left(C_{n}(z)\right)$. For $a(z) \in \mathbb{C}(z)$ let $Q_{n, a(z)}$ be the exact endo-functor of $\mathcal{O}_{c}\left(W_{n}\right)$ that maps an object $M$ to the generalized eigenspace of $D_{n}(z)$ in $M$ with the eigenvalue $a(z)$. The functor

$$
E_{i}(n): \mathcal{O}_{c}\left(W_{n}\right) \rightarrow \mathcal{O}_{c}\left(W_{n-1}\right)
$$

is given by $E_{i}(n)=\bigoplus_{a(z) \in \mathbb{C}(z)} Q_{n-1, a(z) /\left(z-q^{i}\right)} \circ \operatorname{Res}_{b} \circ Q_{n, a(z)}$, where $b$ is chosen as in 4.4 .

4.13. The following result shows that the categorification here of $\mathcal{F}(\mathbf{m})$ arising from the monodromy of the restriction and induction functors is the same as that of [18].

Proposition. For any $i$ and any $n$ there is a natural isomorphism $\operatorname{Res}^{i}(n) \cong E_{i}(n)$.

Proof. We will prove that $\mathrm{KZ}_{n-1} \circ \operatorname{Res}^{i}(n) \cong \mathrm{KZ}_{n-1} \circ E_{i}(n)$, so the result follows from [18, Lemma 2.4]. Let $M \in \mathcal{O}_{c}^{\Delta}\left(W_{n}, \mathfrak{h}\right)$. We first consider $\mathrm{KZ}_{n-1} \circ E_{i}(n)(M)$. By [18, Theorem 2.1], this is the restriction of $\mathrm{KZ}_{n}(M)$ to $\mathcal{H}_{q}\left(W_{n-1}\right)$ followed by the projection onto a block corresponding to the eigenvalue $a(z)$. The blocks of such a restriction are determined by the generalized eigenspaces of $\mathrm{J}_{n-1}$ in $\mathcal{H}_{q}\left(W_{n}\right)$ acting on the restriction: on removing a box $p$ from a multipartition, $\mathrm{J}_{n-1}$ acts by $q^{\text {res }}{ }^{\mathbf{m}}(p)$.

On the other hand $\mathrm{KZ}_{n-1} \circ \operatorname{Res}^{i}(n)(M)$ equals the monodromy of the local system attached to $\operatorname{Res}_{\{1\}}^{W_{n-1}} \circ \operatorname{Res}^{i}(n)(M)$. By Theorem 3.11 this in turn equals the generalized eigenspace of the image of $\Sigma_{M}$ in $B_{n}$ acting on $\mathrm{KZ}_{n}(M)$. We saw in 4.6 that on removing a box $p, \Sigma$ acts by $q^{\mathrm{res}^{\mathbf{m}}(p)+1-n-m_{1}}$. By Lemma 4.14 below, the element $\Sigma$ is mapped to $\sigma_{n-1} \ldots \sigma_{1} \tau \sigma_{1} \ldots \sigma_{n-1}=q^{1-n-m_{1}} \mathrm{~J}_{n-1}$ under the homomorphism $B_{n} \rightarrow \mathcal{H}_{q}\left(W_{n}, \mathfrak{h}\right)$. Thus we have the required equality for objects with $\Delta$ filtrations.

The general case follows since projective objects have $\Delta$-filtrations and $\mathcal{O}_{c}\left(W_{n}, \mathfrak{h}_{n}\right)$ has finite global dimension.

4.14. It remains only to explain the following lemma.

Lemma. Recall the notation $\iota_{W_{1}, W_{2}}$ of 3.11, Let $\psi:\langle\Sigma\rangle \longrightarrow B_{n}$ be the canonical composition

$$
\pi_{1}\left(\mathfrak{h}_{r}^{W_{2}} /\left\langle t_{n}\right\rangle, b\right) \rightarrow \pi_{1}\left(\left(\mathfrak{h}_{r}^{W_{2}} /\left\langle t_{n}\right\rangle \times \mathfrak{h}_{2, r} / W_{n-1}, x_{0}\right) \stackrel{\iota_{\{1\}, W_{n-1}}}{\longrightarrow} \pi_{1}\left(\mathfrak{h}_{r} / N\left(W_{n-1}\right), x_{0}\right) \rightarrow \pi_{1}\left(\mathfrak{h}_{r} / W, x_{0}\right) .\right.
$$

Then $\psi(\Sigma)=\sigma_{n-1} \ldots \sigma_{1} \tau \sigma_{1} \ldots \sigma_{n-1}$.

Proof. If we calculate by lifting paths to $\mathfrak{h}_{\mathrm{r}}$, then a representative of $\psi(\Sigma)$ is given by the path $\gamma_{x_{0}}^{n}$ where for any $1 \leq i \leq n$ we set $\gamma_{x_{0}}^{i}(s)=(1, \ldots, i-1, \exp (2 \pi \sqrt{-1} s) i, i+1, \ldots, n)$ for $s \in[0,1]$. For any $1 \leq i \leq n-1$, set $I_{[i, i+1]}=\left\{\left(a_{1}, a_{2}\right): i \leq\left|a_{1}\right|,\left|a_{2}\right| \leq i+1\right\}$. One can then prove by an unenlightening (for us) calculation in the space $\{1\} \times \cdots \times\{i-1\} \times I_{[i, i+1]} \times\{i+2\} \times\{n\} \subset \mathfrak{h}_{\mathrm{r}}$ that $\sigma_{i} \gamma_{x_{0}}^{i} \sigma_{i}=\gamma_{x_{0}}^{i+1}$. Since $\tau=\gamma_{x_{0}}^{1}$ this confirms the lemma. 
4.15. The KZ-component of the crystal. We end with a short remark on the Cherednik crystal of irreducible representations in $\mathcal{O}_{c}(\mathbb{N})$. Let $\mathbf{B}_{n}=\left\{\lambda \in \operatorname{Irr}\left(W_{n}\right): \mathrm{KZ}_{n}(L(\lambda)) \neq 0\right\}$ and set $\mathbf{B}=$ $\coprod_{n} \mathbf{B}_{n}$. We claim that $\mathbf{B} \cup\{0\}$ is stable under the crystal operators $\tilde{e}_{i}$ and $\tilde{f}_{i}$ for $i \in \mathbb{Z} / e \mathbb{Z}$. Indeed $\tilde{f}_{i} \tilde{e}_{i} b=b$ which ensures that $\operatorname{KZ}\left(\tilde{e}_{i} b\right)=\operatorname{KZ}\left(\operatorname{soc} \operatorname{Res}^{i}\left(\tilde{e}_{i} b\right)\right) \neq 0$, so that $\operatorname{Res}^{i} \operatorname{KZ}\left(\tilde{e}_{i}(b)\right) \cong$ $\operatorname{KZ}\left(\operatorname{Res}^{i}\left(\tilde{e}_{i} b\right)\right) \neq 0$ and so $\operatorname{KZ}\left(\tilde{e}_{i}(b)\right) \neq 0$. The argument for $\tilde{f}_{i} b$ is similar.

By [5, Corollary 5.8] the set $\mathbf{B}$ equals the subset of Uglov multipartitions, that is the subset of multipartitions that label the canonical basis of $L\left(\Lambda_{m_{1}}+\cdots+\Lambda_{m_{\ell}}\right) \subset F(\mathbf{m})$ constructed by Uglov. It follows from the argument in [2, Theorem 6.1] that this crystal equals the crystal defined from Uglov's canonical basis of the Fock space provided that we know that the decomposition matrix of the Hecke algebra is given by the evaluation of Uglov's canonical basis at 1. This is the well-known result of Ariki, [1]. Thus we have an explicit identification of the so-called KZ-component of the crystal with the combinatorial crystal on Uglov multipartitions.

\section{REFERENCES}

[1] S. Ariki, On the decomposition numbers of the Hecke algebra of $G(m, 1, n)$, J. Math. Kyoto Univ. 36 (1996), 789-808. [Page 17]

[2] S. Ariki, Proof of the modular branching rule for cyclotomic Hecke algebras. J. Algebra, 306 (2006), 290-300. [Page 17]

[3] R. Bezrukavnikov and P. Etingof, Parabolic induction and restriction functors for rational Cherednik algebras, Selecta Math. (N.S.) 14 (2009), 397-425. [Pages 3, 4, 6 and 9]

[4] M. Broué, G. Malle, and R. Rouquier. Complex reflection groups, braid groups, Hecke algebras. J. Reine Angew. Math., 500 (1998), 127-190. [Pages 13 and 15]

[5] M. Chlouveraki, I.G. Gordon and S.Griffeth, Canonical basic sets and cell modules for Hecke algebras via Cherednik algebras, Volume 562 of Contemporary Mathematics, American Mathematical Society, 2012, 77-89. [Page 17]

[6] J. Chuang and R. Rouquier. Derived equivalences for symmetric groups and $\mathfrak{s l}_{2}$-categorification. Ann. of Math. (2), 167 (2008), 245-298. [Pages 1] and 11]

[7] C. Dunkl and S. Griffeth. Generalized Jack polynomials and the representation theory of rational Cherednik algebras, Selecta Math. (N.S.), 16 (2010), 791-818. [Page 15]

[8] P. Etingof and V. Ginzburg, Symplectic reflection algebras, Calogero-Moser space, and deformed Harish-Chandra homomorphism, Invent. Math., 147 (2002), 243-348. [Pages 2 and 3]

[9] M. Geck and N. Jacon. Representations of Hecke Algebras at Roots of Unity, volume 15 of Algebra and Applications. Springer, 2011. [Page 16]

[10] V. Ginzburg, N. Guay, E. Opdam, and R. Rouquier. On the category $\mathcal{O}$ for rational Cherednik algebras. Invent. Math., 154 (2003), 617-651. [Pages 104 and 15]

[11] M. Jimbo, K.C. Misra, T. Miwa, M. Okado, Combinatorics of representations of $U_{q}(\hat{\mathfrak{s l}}(n))$ at $q=0$, Comm. Math. Phys., 136 (1991), 543-566. [Pages 1 and 14]

[12] M. Kashiwara and P. Schapira. Moderate and Formal Cohomology associated with Constructible Sheaves, volume 64 of Mémoires Soc. Math. France, 1996. [Page 12]

[13] I. Losev, On isomorphisms of certain functors for Cherednik algebras, arXiv:1011.0211. [Pages 2] and 15]

[14] K. Muraleedaran. Normalizers of Parabolic Subgroups in Unitary Reflection Groups, PhD thesis, University of Sydney, 2005. [Page 8]

[15] Novikov D. and Yakovenko S. Lectures on meromorphic flat connections. NATO Sci. Ser. II Math. Phys. Chem., (137) 387-430, 2004. [Page 9]

[16] E. M. Opdam. Dunkl operators, Bessel functions and the discriminant of a finite Coxeter group. Compositio Math., 85 (1993), 333-373. [Page 9]

[17] R. Rouquier, q-Schur algebras and complex reflection groups, Moscow Math. Journal, 8 (2008), 119-158. [Page 13] 
[18] P. Shan. Crystals of Fock spaces and cyclotomic rational double affine Hecke algebras, Ann. Sci. Éc. Norm. Supér. 44 (2011), 147-182; arXiv:0811.4549. [Pages 121214 and 16]

[19] Wolfgang Walter, Ordinary differential equations, volume 182 of Graduate Texts in Mathematics. SpringerVerlag, New York, 1998. Translated from the sixth German (1996) edition by Russell Thompson, Readings in Mathematics. [Page 9]

I.G.G: School of Mathematics and Maxwell Institute of Mathematics, University of Edinburgh, EDINBURGH, EH9 3JZ, UK

E-mail address: igordon@ed.ac.uk, ohmymo@googlemail.com 